\title{
GRADIENT ESTIMATES OF DIRICHLET HEAT KERNELS FOR UNIMODAL LÉVY PROCESSES
}

\author{
TADEUSZ KULCZYCKI AND MICHAE RYZNAR
}

\begin{abstract}
Under some mild assumptions on the Lévy measure and the symbol we obtain gradient estimates of Dirichlet heat kernels for pure-jump isotropic unimodal Lévy processes in $\mathbb{R}^{d}$.
\end{abstract}

\section{Introduction}

The Dirichlet heat kernels for Lévy processes have been intensively studied in recent years. Qualitatively sharp estimates for classical Dirichlet heat kernels for the Brownian motion were established in 2002 by Zhang 38] for $C^{1,1}$ domains and in 2003 by Varopoulos [34] for Lipschitz domains. Upper bound for the Dirichlet heat kernels for the isotropic stable processes were given in 2006 by Siudeja 31] for convex sets. He used some ideas from [21]. In 2008 Chen, Kim and Song [9] obtained sharp, two sided estimates for the Dirichlet heat kernels for the isotropic stable process for $C^{1,1}$ open sets. In 2009 Bogdan, Grzywny and Ryznar [4] showed similar results for $\kappa$-fat open sets. Gradual extensions were then obtained for some subordinate Brownian motions [6, 7, 8], for Lévy processes with comparable Lévy measure [17] and unimodal Lévy processes satisfying some scaling conditions [3].

The aim of this paper is to obtain gradient estimates of Dirichlet heat kernels for unimodal Lévy processes whose symbols satisfy some scaling conditions and Lévy measures satisfy some regularity conditions. The main result is the following theorem.

Theorem 1.1. Let $X=\left(X_{t}, t \geq 0\right)$ be a pure-jump isotropic Lévy process in $\mathbb{R}^{d}$ with the characteristic exponent $\psi$, which satisfies $\operatorname{WLSC}\left(\underline{\alpha}, \theta_{0}, \underline{C}\right)$ and $\operatorname{WUSC}\left(\bar{\alpha}, \theta_{0}, \bar{C}\right)$ for some $\underline{\alpha}>0, \bar{\alpha} \in(0,2), \theta_{0} \geq 0$, and $\underline{C}, \bar{C}>0$. We assume that the Lévy measure of $X$ is infinite and has the strictly positive density $\nu(x)=\nu(|x|), \nu(r)$ is nonincreasing, absolutely continuous such that $-\nu^{\prime}(r) / r$ is nonincreasing, it satisfies $\nu(r) \leq a \nu(r+1), r \geq 1$ for some constant a.

Let $D \subset \mathbb{R}^{d}$ be an open, nonempty set and $p_{D}(t, x, y)$ be the Dirichlet heat kernel for $X$ on $D$. Then $\nabla_{x} p_{D}(t, x, y)$ exists for any $x, y \in D, t>0$ and we have

$$
\left|\nabla_{x} p_{D}(t, x, y)\right| \leq c\left[\frac{1}{\delta_{D}(x) \wedge 1} \vee \psi^{-}(1 / t)\right] p_{D}(t, x, y), \quad x, y \in D, t \in(0,1],
$$

where $c=c(d, \psi), \delta_{D}(x)=\operatorname{dist}\left(x, D^{c}\right)$ and $\psi^{-}$denotes the generalized inverse of $\psi^{*}(r)=\sup _{\rho \in[0, r]} \psi(\rho)$.

The notation used in the formulation of the above theorem is explained in Preliminaries.

T. Kulczycki was supported in part by the National Science Centre, Poland, grant no. 2015/17/B/ST1/01233, M. Ryznar was supported in part by the National Science Centre, Poland, grant no. 2015/17/B/ST1/01043. 
By the semigroup property one easily gets

Corollary 1.2. If $X$ and $D$ satisfy assumptions of Theorem 1.1 then we have

$$
\left|\nabla_{x} p_{D}(t, x, y)\right| \leq c\left[\frac{1}{\delta_{D}(x) \wedge 1} \vee \psi^{-}(1)\right] p_{D}(t, x, y), \quad x, y \in D, t \in[1, \infty),
$$

where $c=c(d, \psi)$.

Analogous gradient estimates of the classical Dirichlet heat kernel were obtained in 2006 by Zhang [37, Theorem 2.1]. Note that Zhang's estimates have different shape than estimates obtained in Theorem 1.1. Namely, in Zhang's estimate there is an additional term $|x-y| / \sqrt{t}$. This is caused by a different behaviour of Dirichlet heat kernels for pure-jump Lévy processes and the classical one.

The Dirichlet semigroup of the process $X$ on the domain $D \subset \mathbb{R}^{d}$ is given by

$$
P_{t}^{D} f(x)=\int_{D} p_{D}(t, x, y) f(y) d y, \quad x \in D, t>0 .
$$

Directly from Theorem 1.1 we get

Corollary 1.3. Let $X, D$ satisfy assumptions of Theorem 1.1 and $f$ be nonnegative, measurable and bounded on $\mathbb{R}^{d}$. Then $\nabla_{x} P_{t}^{D} f(x)$ exists for any $t>0, x \in D$ and we have

$$
\left|\nabla_{x} P_{t}^{D} f(x)\right| \leq c\left[\frac{1}{\delta_{D}(x) \wedge 1} \vee \psi^{-}(1 / t)\right] P_{t}^{D} f(x), \quad x, y \in D, t \in(0,1],
$$

where $c=c(d, \psi)$.

Note that we have a simple estimate $P_{t}^{D} f(x) \leq P^{x}\left(\tau_{D}>t\right)\|f\|_{\infty}$ and for bounded, sufficiently smooth $\left(C^{1,1}\right)$ domains $D$ sharp estimates of $P^{x}\left(\tau_{D}>t\right)$ are known [3, Theorem 4.5].

Gradient estimates of $P_{t}^{D} f$ for Dirichlet semigroups corresponding to diffusion processes $X$ whose generators are second-order elliptic operators have been intensively studied see e.g. [35, 11], [24, Chapter 3]. The main motivation of such estimates comes from well-known connections with stochastic differential equations see e.g. [16, 33].

Estimates of $\left|\nabla_{x} P_{t} f(x)\right|$, where $\left\{P_{t}\right\}_{t>0}$ is the semigroup of a free Lévy process were obtained in 2012 by Schilling, Sztonyk, Wang [29, Theorem 1.3]. $P_{t} f$ is given by $P_{t} f(x)=\int p_{t}(x-y) f(y) d y$, where $p_{t}(x)$ is the transition density of the process $X$. Interesting estimates of derivatives of $p_{t}$ were obtained in [29] and [15]. The gradient estimates $\left|\nabla_{x} P_{t} f(x)\right|$ from [29] were applied to study stochastic differential equations driven by Lévy processes in [25].

In 2011 estimates of $\left\|D P_{t} f\right\|$ were obtained by Priola and Zabczyk in [27, Theorem 4.14, (5.19)] for the semigroup $\left\{P_{t}\right\}_{t>0}$ of the solution to a nonlinear stochastic partial differential equation

$$
d X_{t}=A X_{t} d t+F\left(X_{t}\right) d t+d Z_{t}
$$

in a real seperable Hilbert space driven by an infinite dimensional, cylindrical stable process $Z$ ( $D$ is here a Fréchet derivative). The results of that paper apply to stochastic heat equation with Dirichlet boundary conditions, see [27, Example 5.5]. It is also worth pointing out that Da Prato, Goldys and Zabczyk in [10, Corollary on 
page 437] obtained estimates of $\left|D P_{t}^{\mathcal{O}} f(x)\right|$ where $\left\{P_{t}^{\mathcal{O}}\right\}_{t>0}$ is the Dirichlet semigroup of the process $X$ on $\mathcal{O}, X$ is the solution of a linear stochastic equation

$$
d X=A X d t+d W
$$

in a separable Hilbert space $H$ driven by a Wiener process $W$ on $H$, and $\mathcal{O}$ is an open subset of $H$.

The paper is organized as follows. In Section 2 we introduce the notation and collect known facts needed in the sequel. In Section 3 we provide some auxiliary estimates of the heat kernel and the Lévy measure. The main section of the paper is Section 4 in which we prove Theorem 1.1. The next section contains examples of processes which satisfy assumptions of Theorem 1.1. In Appendix we provide a proof of Theorem 2.5 which is an extension of Theorem 4.5 in [3].

\section{Preliminaries}

In the whole paper we use a convention that for a radial function $f: \mathbb{R}^{d} \rightarrow \mathbb{R}$ we write $f(x)=f(r)$, if $x \in \mathbb{R}^{d}$ and $|x|=r$. All constants appearing in this paper are positive and finite. We write $\kappa=\kappa(a, \ldots, z)$ to emphasize that $\kappa$ depends only on $a, \ldots, z$. We adopt the convention that constants denoted by $c$ (or $c_{1}, c_{2}$ ) may change their value from one use to another. We write $f(x) \approx g(x)$ for $x \in A$ and say $f$ and $g$ are comparable for $x \in A$ if $f, g \geq 0$ on $A$ and there is a number $c \geq 1$, called comparability constant, such that $c^{-1} f(x) \leq g(x) \leq c f(x)$ for $x \in A$. For $x \in \mathbb{R}^{d}$ and $r>0$ we let $B(x, r)=\left\{y \in \mathbb{R}^{d}:|y-x|<r\right\}$. We denote $a \wedge b=\min (a, b)$ and $a \vee b=\max (a, b)$ for $a, b \in \mathbb{R}$. When $D \subset \mathbb{R}^{d}$ is an open set we denote by $\mathcal{B}(D)$ a family of Borel subsets of $D$.

A Borel measure on $\mathbb{R}^{d}$ is called isotropic unimodal if on $\mathbb{R}^{d} \backslash\{0\}$ it is absolutely continuous with respect to the Lebesgue measure and has a finite, radial, radially nonincreasing density function. Such measures may have an atom at the origin. A Lévy process $X=\left(X_{t}, t \geq 0\right)$ is called isotropic unimodal if its transition probability $p_{t}(d x)$ is isotropic unimodal for all $t>0$. Unimodal isotropic purejump Lévy processes are characterized [36] by unimodal isotropic Lévy measures $\nu(d x)=\nu(x) d x=\nu(|x|) d x$.

The characteristic exponent of $X$ is given by

$$
\psi(\xi)=\int_{\mathbb{R}^{d}}(1-\cos \langle\xi, x\rangle) \nu(d x), \quad \xi \in \mathbb{R}^{d} .
$$

In the whole paper we assume that $X$ is a pure-jump isotropic unimodal Lévy process in $\mathbb{R}^{d}$ with the characteristic exponent $\psi$ and that the Lévy measure of $X$ is infinite. We also assume that the following Hartman-Wintner condition holds

$$
\lim _{|x| \rightarrow \infty} \frac{\psi(x)}{\log |x|}=\infty
$$

This guarantees that for any $t>0, p_{t}(d x)$ has a radial, radially nonincreasing density function $p_{t}(x)$, which is bounded and smooth on $\mathbb{R}^{d}$.

The derivative $\nu^{\prime}(r)$ is understood as a function (defined a.e. on $(0, \infty)$ ) such that $\nu(r)=-\int_{r}^{\infty} \nu^{\prime}(\rho) d \rho, r>0$. In fact, under the assumption that $-\nu^{\prime}(r) / r$ is nonincreasing on the set where it is defined, we can always take a version which is well defined for each point $r>0$ and $-\nu^{\prime}(r) / r$ is nonincreasing on $(0, \infty)$. Throughout the whole paper we use that meaning of $\nu^{\prime}(r)$. Note also that if $\nu(r)$ is convex then $-\nu^{\prime}(r) / r$ is nonincreasing (in the above sense). 
Now we recall the definition of scaling conditions (cf. [2]). Let $\varphi$ be a nonnegative, non-zero function on $[0, \infty)$. We say that $\varphi$ satisfies a weak lower scaling condition $\operatorname{WLSC}\left(\underline{\alpha}, \theta_{0}, \underline{C}\right)$ (and write $\varphi \in \operatorname{WLSC}\left(\underline{\alpha}, \theta_{0}, \underline{C}\right)$ or $\varphi \in \mathrm{WLSC}$ ) if there are numbers $\underline{\alpha}>0, \theta_{0} \geq 0$ and $\underline{C}>0$ such that

$$
\varphi(\lambda \theta) \geq \underline{C} \lambda \underline{\alpha}^{\varphi}(\theta), \quad \text { for } \quad \lambda \geq 1, \theta \geq \theta_{0} .
$$

We say that $\varphi$ satisfies a weak upper scaling condition $\operatorname{WUSC}\left(\bar{\alpha}, \theta_{0}, \bar{C}\right)$ (and write $\varphi \in \operatorname{WUSC}\left(\underline{\alpha}, \theta_{0}, \underline{C}\right)$ or $\varphi \in$ WUSC $)$ if there are numbers $\bar{\alpha} \in(0,2), \theta_{0} \geq 0$ and $\bar{C}>0$ such that

$$
\varphi(\lambda \theta) \leq \bar{C} \lambda^{\bar{\alpha}} \varphi(\theta), \quad \text { for } \quad \lambda \geq 1, \theta \geq \theta_{0} .
$$

Note that the condition $\psi \in \operatorname{WLSC}\left(\underline{\alpha}, \theta_{0}, \underline{C}\right)$ implies (2) $)$.

Recall that the maximal characteristic function is defined by $\psi^{*}(r)=\sup _{\rho \in[0, r]} \psi(\rho)$, $r \geq 0$. We define its generalized inverse $\psi^{-}:[0, \infty) \rightarrow[0, \infty]$ by

$$
\psi^{-}(x)=\inf \left\{y \geq 0: \psi^{*}(y) \geq x\right\}, \quad 0 \leq x<\infty,
$$

with the convention that $\inf \emptyset=\infty$. It is well known [2, Proposition 2] (see also [12, Proposition 1])

$$
\psi(r) \leq \psi^{*}(r) \leq \pi^{2} \psi(r), \quad r \geq 0 .
$$

In the paper we will use the renewal function $V$ of the properly normalized ascending ladder-height process of $X_{t}^{(1)}$, where $X_{t}^{(1)}$ is the first coordinate of $X_{t}$. The ladder-height process is a subordinator with the Laplace exponent

$$
\kappa(\xi)=\exp \left\{\frac{1}{\pi} \int_{0}^{\infty} \frac{\log \psi(\xi \zeta)}{1+\zeta^{2}} d \zeta\right\}, \quad \xi \geq 0,
$$

and $V(x)$ is its potential measure of the half-line $(-\infty, x)$. The Laplace transform of $V$ is given by

$$
\int_{0}^{\infty} V(x) e^{-\xi x} d x=\frac{1}{\xi \kappa(\xi)}, \quad \xi>0
$$

For a detailed discussion of the properties of $V$ we refer the reader to [30]. We have $V(x)=0$ for $x \leq 0$ and $V(\infty):=\lim _{r \rightarrow \infty} V(r)=\infty$. $V$ is subadditive, that is

$$
V(x+y) \leq V(x)+V(y), \quad x, y \in \mathbb{R} .
$$

It is known that $V$ is absolutely continuous on $(0, \infty)$ and strictly increasing on $(0, \infty)$. We will use $V$ and its inverse function $V^{-1}$ in the estimates of heat kernels. By [1, Proof of Proposition 2.4] we have

$$
c_{1} \psi\left(\frac{1}{r}\right) \leq \frac{1}{V^{2}(r)} \leq c_{2} \psi\left(\frac{1}{r}\right), \quad r>0
$$

where $c_{1}, c_{2}$ are absolute constants. It is clear that

$$
r<V^{-1}(\sqrt{t}) \Longleftrightarrow V^{2}(r)<t, \quad r, t>0,
$$

and

$$
r<V^{-1}(\sqrt{t}) \Longleftrightarrow \frac{t}{V^{2}(r) r^{d}}>\left[V^{-1}(\sqrt{t})\right]^{-d}, \quad r, t>0 .
$$

If $\psi \in$ WLSC and $\psi \in$ WUSC then by (3), (4) we get for any $t \in(0,1]$

$$
\frac{1}{V^{-1}(\sqrt{t})} \approx \psi^{-}\left(\frac{1}{t}\right)
$$

where the comparability constant depends only on $\psi$. 
By (4), [3, (1.9)], 3, Remark 1.4] and arguments similar to the justification of [3, (1.8)] we obtain the following scaling properties $V^{-1}$.

Lemma 2.1. Let $\psi$ satisfy $W L S C\left(\underline{\alpha}, \theta_{0}, \underline{C}\right)$ and $W U S C\left(\bar{\alpha}, \theta_{0}, \bar{C}\right)$ for some $\underline{\alpha}>0$, $\bar{\alpha} \in(0,2), \theta_{0} \geq 0$, and $\underline{C}, \bar{C}>0$. Then there exists $c_{1}=c_{1}(d, \psi)$ such that

$$
V^{-1}(\eta \omega) \geq c_{1} \eta^{2 / \underline{\alpha}} V^{-1}(\omega), \quad \text { for } \quad \eta \in(0,1], \omega \in(0,1],
$$

Now we introduce the condition $(\mathbf{H})$, the reader is referred to [3] for a detailed exposition.

Definition 2.2. We say that condition $(\mathbf{H})$ holds if for every $r>0$ there is $H_{r} \geq 1$ such that

$$
V(z)-V(y) \leq H_{r} V^{\prime}(x)(z-y) \quad \text { whenever } \quad 0<x \leq y \leq z \leq 5 x \leq 5 r .
$$

We may assume that $r \rightarrow H_{r}$ is nondecreasing. It is known [1, Section 7.1] that if $\psi \in$ WLSC and $\psi \in$ WUSC then $(\mathbf{H})$ holds.

As usual for any $x \in \mathbb{R}^{d}$ we denote by $E^{x}, P^{x}$ the expectation and the probability measure for the process starting from $x$. Let $D \subset \mathbb{R}^{d}$ be an open, nonempty set. By $\tau_{D}=\inf \left\{t \geq 0: X_{t} \notin D\right\}$ we denote the first exit time of the process $X$ from $D$. We define a killed process $X_{t}^{D}$ by $X_{t}^{D}=X_{t}$ if $t<\tau_{D}$ and $X_{t}^{D}=\partial$ otherwise, where $\partial$ is some point adjoined to $D$. The transition density for $X_{t}^{D}$ on $D$ is given by

$$
p_{D}(t, x, y)=p_{t}(x-y)-E^{x}\left(p_{t-\tau_{D}}\left(y-X\left(\tau_{D}\right)\right), \tau_{D}<t\right), \quad t>0, x, y \in D .
$$

$p_{D}(t, x, y)$ is called the Dirichlet heat kernel for the process $X$ on the set $D$. For any $t>0$ we put $p_{D}(t, x, y)=0$ if $x \notin D$ or $y \notin D$. It is well known that $p_{D}(t, x, y)=p_{D}(t, y, x)$ for any $t>0, x, y \in D$. The Dirichlet semigroup $\left\{P_{t}^{D}\right\}_{t>0}$ of $X$ on an open set $D \subset \mathbb{R}^{d}$ is defined by

$$
P_{t}^{D} f(x)=E^{x}\left(f\left(X_{t}\right), \tau_{D}>t\right), \quad x \in D,
$$

for any measurable, bounded function $f: D \rightarrow \mathbb{R}$. It is well known that $P_{t}^{D} f(x)=$ $\int_{D} p_{D}(t, x, y) f(y) d y, x \in D, t>0$.

The corresponding Green function is defined by

$$
G_{D}(x, y)=\int_{0}^{\infty} p_{D}(t, x, y) d t, \quad x, y \in D, \quad x \neq y,
$$

$G_{D}(x, x)=\infty, x \in D, G_{D}(x, y)=0$ if $x \notin D$ or $y \notin D$.

Let $D \subset \mathbb{R}^{d}$ be a bounded, open, nonempty set. The distribution $P^{x}\left(X\left(\tau_{D}\right) \in \cdot\right)$ is called the harmonic measure with respect to $X$. The harmonic measure for Borel sets $A \subset(\bar{D})^{c}$ is given by the Ikeda-Watanabe formula [14]

$$
P^{x}\left(X\left(\tau_{D}\right) \in A\right)=\int_{A} \int_{D} G_{D}(x, y) \nu(y-z) d y d z, \quad x \in D .
$$

When $D \subset \mathbb{R}^{d}$ is a bounded, open Lipschitz set then we have [32, 26]

$$
P^{x}\left(X\left(\tau_{D}\right) \in \partial D\right)=0, \quad x \in D .
$$

It follows that for such sets $D$ the Ikeda-Watanabe formula (9) holds for any Borel set $A \subset D^{c}$. Let $D \subset \mathbb{R}^{d}$ be a bounded, open, nonempty set. For any $s>0, x \in D$, $z \in(\bar{D})^{c}$ put

$$
h_{D}(x, s, z)=\int_{D} p_{D}(s, x, y) \nu(y-z) d y .
$$


By Ikeda-Watanabe formula [14] and standard arguments (see e.g. [21, proof of Proposition 2.5]) for any Borel sets $A \subset(0, \infty), B \subset(\bar{D})^{c}$ we have

$$
P^{x}\left(\tau_{D} \in A, X\left(\tau_{D}\right) \in B\right)=\int_{A} \int_{B} h_{D}(x, s, z) d z d s, \quad x \in D .
$$

If (10) holds then we can take $B \subset D^{c}$ in (12).

The main tool used in the proof of Theorem 1.1 is the so-called difference process already constructed in [20] (cf. also [19]). For the Reader convenience we recall its definition and basic properties from [20, Section 4]. We will use the following notation $\hat{x}=\left(-x_{1}, x_{2}, \ldots, x_{d}\right)$ for $x=\left(x_{1}, x_{2}, \ldots, x_{d}\right), D_{+}=\left\{\left(x_{1}, x_{2}, \ldots, x_{d}\right) \in\right.$ $\left.D: x_{1}>0\right\}, D_{-}=\left\{\left(x_{1}, x_{2}, \ldots, x_{d}\right) \in D: x_{1}<0\right\}$ for $D \subset \mathbb{R}^{d}$. For any $t>0$, $x, y \in \mathbb{R}_{+}^{d}$ put

$$
\tilde{p}_{t}(x, y)=p_{t}(x-y)-p_{t}(\hat{x}-y) .
$$

Now let us define $\tilde{P}_{t}(x, A), t \geq 0, x \in \mathbb{R}_{+}^{d}, A \in \mathcal{B}\left(\mathbb{R}_{+}^{d}\right)$ by $\tilde{P}_{t}(x, A)=\int_{A} \tilde{p}_{t}(x, y) d y$, $t>0$, and $\tilde{P}_{0}(x, \cdot)=\delta_{x}$. Let us augment $\mathbb{R}_{+}^{d}$ by an extra point $\partial$ so that $\mathbb{R}_{+}^{d} \cup\{\partial\}$ is a one-point compactification of $\mathbb{R}_{+}^{d}$. We extend $\tilde{P}_{t}(x, A)$ to a Markov transition function on $\mathbb{R}_{+}^{d} \cup\{\partial\}$ by setting

$$
\tilde{P}_{t}(x, A)= \begin{cases}\tilde{P}_{t}\left(x, A \cap \mathbb{R}_{+}^{d}\right)+1_{A}(\partial)\left(1-\tilde{P}_{t}\left(x, \mathbb{R}_{+}^{d}\right)\right), & \text { for } \quad x \in \mathbb{R}_{+}^{d}, \\ 1_{A}(\partial), & \text { for } \quad x=\partial\end{cases}
$$

for any $A \subset \mathbb{R}_{+}^{d} \cup\{\partial\}$ which is in the $\sigma$-algebra in $\mathbb{R}_{+}^{d} \cup\{\partial\}$ generated by $\mathcal{B}\left(\mathbb{R}_{+}^{d}\right)$. Then [20, Section 4] there exists a Hunt process $\tilde{X}=\left(\tilde{X}_{t}, t \geq 0\right)$ with the state space $\mathbb{R}_{+}^{d} \cup\{\partial\}$ and the transition function $\tilde{P}_{t}(x, A)$. We call it the difference process. We will denote by $\tilde{P}^{x}, \tilde{E}^{x}$ the probability and the expected value with respect to the process $\tilde{X}$ starting from $x$.

We say that $D \subset \mathbb{R}^{d}$ satisfies the outer cone condition if for any $z \in \partial D$ there exist $r>0$ and a cone $A$ with vertex $z$ such that $A \cap B(z, r) \subset D^{c}$.

Let $D \subset \mathbb{R}_{+}^{d}$ be an open, nonempty set satisfying the outer cone condition. For any $t>0, x, y \in D$ we put

$$
\tilde{p}_{D}(t, x, y)=\tilde{p}_{t}(x, y)-\tilde{E}^{x}\left(\tilde{p}_{t-\tau_{D}}\left(\tilde{X}\left(\tau_{D}\right), y\right), t>\tau_{D}\right),
$$

where $\tau_{D}=\inf \left\{t \geq 0: \tilde{X}_{t} \notin D\right\}$. For any Borel $A \subset D, x \in D$ and $t>0$ we have

$$
\tilde{P}^{x}\left(\tilde{X}_{t} \in A, \tau_{D}>t\right)=\int_{A} \tilde{p}_{D}(t, x, y) d y .
$$

We say that a set $D \subset \mathbb{R}^{d}$ is symmetric if for any $x \in D$ we have $\hat{x} \in D$.

Let $D \subset \mathbb{R}^{d}$ be an open, nonempty, symmetric set satisfying the outer cone condition. For any $t>0, x, y \in D_{+}$, we have

$$
\tilde{p}_{D_{+}}(t, x, y)=p_{D}(t, x, y)-p_{D}(t, \hat{x}, y) \text {. }
$$

It follows that

$$
0 \leq p_{D}(t, x, y)-p_{D}(t, \hat{x}, y) \leq p_{t}(x-y)-p_{t}(\hat{x}-y)
$$

for any $t>0, x, y \in D_{+}$. We define the Green function for $\tilde{X}_{t}$ and $D_{+}$by

$$
\tilde{G}_{D_{+}}(x, y)=\int_{0}^{\infty} \tilde{p}_{D_{+}}(t, x, y) d t, \quad x, y \in D_{+},
$$


$\tilde{G}_{D_{+}}(x, x)=\infty, x \in D_{+}, \tilde{G}_{D_{+}}(x, y)=0$ if $x \notin D_{+}$or $y \notin D_{+}$. For any $x, y \in D_{+}$, $x \neq y$ and a Borel set $A \subset \mathbb{R}_{+}^{d}$ put

$$
\tilde{\nu}(x, y)=\lim _{t \rightarrow 0} \frac{\tilde{p}_{t}(x, y)}{t}=\nu(x-y)-\nu(\hat{x}-y)
$$

and $\tilde{\nu}(x, A)=\int_{A} \tilde{\nu}(x, y) d y$. We call $\tilde{\nu}(x, A)$ the Lévy measure for the process $\tilde{X}$.

If $D \subset \mathbb{R}^{d}$ is a symmetric, open, nonempty, bounded Lipschitz set then for a Borel set $B \subset \mathbb{R}_{+}^{d} \backslash D$ and $x \in D_{+}$we have

$$
\tilde{P}^{x}\left(\tilde{X}\left(\tau_{D_{+}}\right) \in B\right)=\int_{D_{+}} \tilde{G}_{D_{+}}(x, y) \int_{B} \tilde{\nu}(y, z) d z d y .
$$

For any $s>0, x \in D_{+}, z \in \mathbb{R}_{+}^{d} \backslash \overline{D_{+}}$put

$$
\tilde{h}_{D}(x, s, z)=\int_{D_{+}} \tilde{p}_{D_{+}}(s, x, y) \tilde{\nu}(y, z) d y .
$$

By (15) and standard arguments (see e.g. [21, proof of Proposition 2.5]) for any Borel sets $A \subset(0, \infty), B \subset \mathbb{R}_{+}^{d} \backslash D_{+}$we have

$$
\tilde{P}^{x}\left(\tau_{D} \in A, \tilde{X}\left(\tau_{D}\right) \in B\right)=\int_{A} \int_{B} \tilde{h}_{D}(x, s, z) d z d s, \quad x \in D_{+} .
$$

Lemma 5.2 in [20] gives

Lemma 2.3. Let $X$ be a process which satisfies assumptions of Theorem 1.1. Then for any $v, z \in \mathbb{R}_{+}^{d}$ we have

$$
\tilde{\nu}(v, z) \leq c|z-\hat{z}| \frac{\nu(v-z)}{1 \wedge|v-z|}\left(1+\frac{|v-\hat{z}|}{|v-z|}\right)
$$

where $c=c(d, \psi)$.

For any open bounded set $D \subset \mathbb{R}^{d}$ and any $t>0$ the operators $P_{t}^{D}$ (defined by (8)) acting on $L^{2}(D)$ are Hilbert-Schmidt operators. From general theory of semigroups it is well known that there exists a sequence of eigenvalues $0<\lambda_{1}^{D}<\lambda_{2}^{D} \leq \lambda_{3}^{D} \leq \ldots$, $\lim _{n \rightarrow \infty} \lambda_{n}^{D}=\infty$ and an orthonormal basis of eigenfunctions $\left\{\varphi_{n}^{D}\right\}_{n=1}^{\infty}$ such that

$$
P_{t}^{D} \varphi_{n}^{D}(x)=\exp \left(-\lambda_{n}^{D} t\right) \varphi_{n}^{D}(x), \quad t>0, x \in D, n \in \mathbb{N} .
$$

The following lemma was proved in [3].

Lemma 2.4. Let $D$ be an open bounded set containing a ball of radius $R>0$. Then

$$
\frac{1}{8}\left(\frac{R}{\operatorname{diamD}}\right)^{2} \leq \lambda_{1}^{D} V^{2}(R) \leq c\left(\frac{\operatorname{diamD}}{R}\right)^{d / 2},
$$

where $c=c(d)$.

For $R>0$, by Lemma 2.4, we get

$$
\lambda_{1}^{B(0, R)} \approx \frac{1}{V^{2}(R)},
$$

where the comparability constant depends only on $d$.

The following result is the partial extension of Theorem 4.5 in [3]. Recall that for any set $D \subset \mathbb{R}^{d}$ and $x \in \mathbb{R}^{d}, \delta_{D}(x)=\operatorname{dist}\left(x, D^{c}\right)$. 
Theorem 2.5. Let $R>0$ and put $\lambda_{1}(R)=\lambda_{1}^{B(0, R)}$. If $\psi \in \operatorname{WLSC}\left(\underline{\alpha}, \theta_{0}, \underline{C}\right) \cap$ $\operatorname{WUSC}\left(\bar{\alpha}, \theta_{0}, \bar{C}\right)$, for some $\underline{\alpha}>0, \bar{\alpha} \in(0,2), \theta_{0} \geq 0, \underline{C}, \bar{C}>0$ and the Lévy measure has strictly positive density then we have

$$
\begin{aligned}
& c_{1}^{-1} \mathbb{P}^{x}\left(\tau_{B(0, R)}>\frac{t}{2}\right) \mathbb{P}^{y}\left(\tau_{B(0, R)}>\frac{t}{2}\right) p_{t \wedge V^{2}(R)}(x-y) \\
\leq & p_{B(0, R)}(t, x, y) \leq c_{1} \mathbb{P}^{x}\left(\tau_{B(0, R)}>\frac{t}{2}\right) \mathbb{P}^{y}\left(\tau_{B(0, R)}>\frac{t}{2}\right) p_{t \wedge V^{2}(R)}(x-y)
\end{aligned}
$$

and

$c_{2}^{-1} e^{-\lambda_{1}(R) t}\left(\frac{V\left(\delta_{B(0, R)}(x)\right)}{\sqrt{t} \wedge V(R)} \wedge 1\right) \leq \mathbb{P}^{x}\left(\tau_{B(0, R)}>t\right) \leq c_{2} e^{-\lambda_{1}(R) t}\left(\frac{V\left(\delta_{B(0, R)}(x)\right)}{\sqrt{t} \wedge V(R)} \wedge 1\right)$

for all $x, y \in B(0, R)$ and $t>0$. The constants $c_{1}, c_{2}$ depend on $R, d$ and $\psi$. They are nondecreasing with respect to $R$.

Let $R \in(0,1]$. An immediate corollary of Theorem 2.5, (17) and subadditivity of $V$ is the following comparability

$$
p_{B(0, R)}(t, x, y) \approx p_{t}(x-y), \quad t \leq V^{2}(R), x, y \in B(0,3 R / 4) .
$$

The comparability constant depends only on $d$ and $\psi$.

\section{Auxiliary estimates of the heat kernel and the LÉvy measure}

In this section we present some estimates of $p_{t}(r)$ and $\nu(r)$ which will be needed in the sequel.

The following estimate follows from [13, Corollary 2.12].

Lemma 3.1. For any $r, t>0$ we have

$$
p_{t}(r) \geq c t \nu(r) \exp \left(\frac{-c_{1} t}{V^{2}(r)}\right),
$$

where $c=c(d), c_{1}=c_{1}(d)$.

The next lemma follows from [2, Corollary 7].

Lemma 3.2. For any $r, t>0$ we have

$$
p_{t}(r) \leq\left(p_{t}(0) \wedge \frac{c t}{V^{2}(r) r^{d}}\right)
$$

where $c=c(d)$.

The next two lemmas are easy consequences of the results from [2] and [3].

Lemma 3.3. Let $\psi$ satisfy $W L S C\left(\underline{\alpha}, \theta_{0}, \underline{C}\right)$ for some $\underline{\alpha}>0, \theta_{0} \geq 0$, and $\underline{C}>0$. Then for any $T>0$ there exists $c=c(d, \psi, T)$ such that for any $t \in(0, T]$ we have

$$
p_{t}(0) \leq c\left[V^{-1}(\sqrt{t})\right]^{-d} .
$$

Proof. By [3, Lemma 1.6] there exists $T_{0}=T_{0}(d, \psi)$ and $c_{1}=c_{1}(d, \psi)$ such that for any $t \in\left(0, T_{0}\right]$ we have

$$
p_{t}(0) \leq c_{1}\left[V^{-1}(\sqrt{t})\right]^{-d} .
$$

On the other hand for any $t \in\left(T_{0}, T\right]$ we have

$$
p_{t}(0) \leq p_{T_{0}}(0) \leq p_{T_{0}}(0) \frac{\left[V^{-1}(\sqrt{T})\right]^{d}}{\left[V^{-1}(\sqrt{t})\right]^{d}} .
$$


Lemma 3.4. Let $\psi$ satisfy $W L S C\left(\underline{\alpha}, \theta_{0}, \underline{C}\right)$ and $W U S C\left(\bar{\alpha}, \theta_{0}, \bar{C}\right)$ for some $\underline{\alpha}>0$, $\bar{\alpha} \in(0,2), \theta_{0} \geq 0$, and $\underline{C}, \bar{C}>0$. We assume also that the Lévy measure has strictly positive density. Then for any $R_{0}>0$ there exist $c_{1}=c_{1}\left(d, \psi, R_{0}\right), c_{2}=c_{2}(d)$ such that for any $r \in\left(0, R_{0}\right]$ we have

$$
\frac{c_{1}}{V^{2}(r) r^{d}} \leq \nu(r) \leq \frac{c_{2}}{V^{2}(r) r^{d}}
$$

Proof. The upper bound follows from Lemma 3.2 .

By [2, Corollary 22] and (44) there exists $r_{0}=r_{0}(d, \psi)$ and $c_{1}=c_{1}(d, \psi)$ such that for any $r \in\left(0, r_{0}\right]$ we have

$$
\nu(r) \geq \frac{c_{1}}{V^{2}(r) r^{d}}
$$

Hence, for any $r \in\left(r_{0}, R_{0}\right]$ we obtain

$$
\nu(r) \geq \nu\left(R_{0}\right) \geq \nu\left(R_{0}\right) \frac{V^{2}\left(r_{0}\right) r_{0}^{d}}{V^{2}(r) r^{d}}
$$

Remark 3.5. Note that under the assumptions of Lemma 3.4, for any $R>0$, there exists $c=c(d, \psi, R)$ such that the Lévy measure satisfies $\nu(r) \leq c \nu(2 r)$ for any $0<r \leq R$.

Lemma 3.6. Let $\psi$ satisfy $W L S C\left(\underline{\alpha}, \theta_{0}, \underline{C}\right)$ and $W U S C\left(\bar{\alpha}, \theta_{0}, \bar{C}\right)$ for some $\underline{\alpha}>0$, $\bar{\alpha} \in(0,2), \theta_{0} \geq 0$, and $\underline{C}, \bar{C}>0$. We assume also that the Lévy measure has strictly positive density. Fix $R>0$. If $t \in\left(0,1 \vee V^{2}(R)\right], r>0$ and $r<V^{-1}(\sqrt{t})$ then

$$
p_{t}(r) \approx\left[V^{-1}(\sqrt{t})\right]^{-d},
$$

if $t>0, r \in[0, R]$ and $r \geq V^{-1}(\sqrt{t})$ then

$$
p_{t}(r) \approx \frac{t}{V^{2}(r) r^{d}}
$$

For any $t \in\left(0,1 \vee V^{2}(R)\right], r \in[0, R]$ we have

$$
p_{t}(r) \approx \min \left\{\left[V^{-1}(\sqrt{t})\right]^{-d}, \frac{t}{V^{2}(r) r^{d}}\right\} .
$$

The comparability constants depend only on $d, \psi$ and $R$.

Proof. In view of (6) it is enough to show (19) and (20).

Case 1. $r<V^{-1}(\sqrt{t}), t \in\left(0,1 \vee V^{2}(R)\right], r>0$.

By the fact that $r \rightarrow p_{t}(r)$ is nonincreasing and Lemma 3.1 we get

$$
p_{t}(r) \geq p_{t}\left(V^{-1}(\sqrt{t})\right) \geq c t \nu\left(V^{-1}(\sqrt{t})\right) \exp \left(\frac{-c_{1} t}{V^{2}\left(V^{-1}(\sqrt{t})\right)}\right) .
$$

Note that $V^{2}\left(V^{-1}(\sqrt{t})\right)=t$. Using this and Lemma 3.4 (applied for $R_{0}=R \vee$ $\left.V^{-1}(1)\right)$ we get that the right hand side of (22) is bounded from below by

$$
\frac{c t}{V^{2}\left(V^{-1}(\sqrt{t})\right)\left[V^{-1}(\sqrt{t})\right]^{d}}=c\left[V^{-1}(\sqrt{t})\right]^{-d} .
$$


On the other hand, by Lemma 3.2 and Lemma 3.3 (applied for $T=1 \vee V^{2}(R)$ ) we get

$$
p_{t}(r) \leq p_{t}(0) \leq c\left[V^{-1}(\sqrt{t})\right]^{-d} .
$$

Case 2. $r \geq V^{-1}(\sqrt{t}), t>0, r \in[0, R]$.

Note that by (5) we have $t / V^{2}(r) \leq 1$. Using this, Lemma 3.1 and Lemma 3.4 we get

$$
p_{t}(r) \geq c t \nu(r) \exp \left(\frac{-c_{1} t}{V^{2}(r)}\right) \geq \frac{c t}{V^{2}(r) r^{d}} .
$$

By Lemma 3.2 we obtain

$$
p_{t}(r) \leq \frac{c t}{V^{2}(r) r^{d}}
$$

In the sequel we need estimates of $\frac{d}{d r} p_{t}(r)$. They are based on the following result.

Theorem 3.7. [20, Theorem 1.5] Let $X$ be a pure-jump isotropic Lévy process in $\mathbb{R}^{d}$ with the characteristic exponent $\psi$. We assume that its Lévy measure is infinite and has the density $\nu(x)=\nu(|x|)$ such that $\nu(r)$ is nonincreasing, absolutely continuous and $-\nu^{\prime}(r) / r$ is nonincreasing. We denote transition densities of $X$ by $p_{t}(x)=p_{t}(|x|)$. Then there exists a Lévy process $X_{t}^{(d+2)}$ in $\mathbb{R}^{d+2}$ with the characteristic exponent $\psi^{(d+2)}(\xi)=\psi(|\xi|), \xi \in \mathbb{R}^{d+2}$ and the radial, radially nonincreasing transition density $p_{t}^{(d+2)}(x)=p_{t}^{(d+2)}(|x|)$ satisfying

$$
p_{t}^{(d+2)}(r)=\frac{-1}{2 \pi r} \frac{d}{d r} p_{t}(r), \quad r>0 .
$$

Moreover, $p_{t}^{(d+2)}$ is continuous at any $x \neq 0$.

Lemma 3.8. Let $X$ satisfy assumptions of Theorem 1.1. Then the assumptions of Theorem 3.7 are satisfied. Denote by $X_{t}^{(d+2)}, \nu^{(d+2)}(x), \psi^{(d+2)}(x)$ the corresponding process in $\mathbb{R}^{d+2}$, the density of its Lévy measure and its characteristic exponent, respectively. Then $X_{t}^{(d+2)}$ is a pure-jump isotropic unimodal Lévy process in $\mathbb{R}^{d+2}$, its Lévy measure is infinite and satisfies

$$
\forall R>0 \quad \nu^{(d+2)}(R)>0 .
$$

$\psi^{(d+2)}$ satisfies $\operatorname{WLSC}\left(\underline{\alpha}, \theta_{0}, \underline{C}\right)$ and $\operatorname{WUSC}\left(\bar{\alpha}, \theta_{0}, \bar{C}\right)$.

Proof. The assumptions of Theorem 3.7 are clearly satisfied. The fact that $X_{t}^{(d+2)}$ is a pure-jump isotropic unimodal Lévy process in $\mathbb{R}^{d+2}$ follows directly from Theorem 3.7. The properties of $\psi^{(d+2)}$ are also clear because $\psi^{(d+2)}(|x|)=\psi(|x|)$. The fact that the Lévy measure of $X_{t}^{(d+2)}$ is infinite is stated in [20, proof of Theorem 1.5]. Now we will justify (24). Since $X_{t}^{(d+2)}$ is isotropic unimodal we know that $r \rightarrow \nu^{(d+2)}(r)$ is nonincreasing. By [20, proof of Theorem 1.5] we have

$$
\nu^{(d+2)}(r)=\frac{-1}{2 \pi r} \frac{d \nu}{d r}(r), \quad r>0 .
$$

If $\nu^{(d+2)}(R)=0$ for some $R>0$ then $\nu^{(d+2)}(r)=0$ for all $r \geq R$. But then we would have $\nu(r)=0$ for all $r \geq R$ which contradicts assumptions in Theorem 1.1.

Using Theorem 3.7 and Lemmas 3.8, 3.6 we obtain 
Lemma 3.9. Let $X$ satisfy assumptions of Theorem [1.1. Fix $R>0$. If $t \in(0,1]$, $r>0$ and $r<V^{-1}(\sqrt{t})$ then

$$
\left|\frac{d}{d r} p_{t}(r)\right| \approx r\left[V^{-1}(\sqrt{t})\right]^{-d-2},
$$

if $t>0, r \in(0, R]$ and $r \geq V^{-1}(\sqrt{t})$ then

$$
\left|\frac{d}{d r} p_{t}(r)\right| \approx \frac{t}{V^{2}(r) r^{d+1}}
$$

For any $t \in(0,1], r \in(0, R]$ we have

$$
\left|\frac{d}{d r} p_{t}(r)\right| \approx r \min \left\{\left[V^{-1}(\sqrt{t})\right]^{-d-2}, \frac{t}{V^{2}(r) r^{d+2}}\right\} .
$$

The comparability constants depend only on $d, \psi$ and $R$.

\section{Gradient estimates}

In this section we prove Theorem 1.1. In the whole section we suppose that the process $X$ satisfies assumptions of this theorem.

Lemma 4.1. For any $t \in(0,1], r \in(0,2]$ we have

$$
\left|\frac{d}{d r} p_{t}(r)\right| \leq c\left(\frac{p_{t}(r)}{r} \wedge \frac{p_{t}(r)}{V^{-1}(\sqrt{t})}\right)
$$

where $c=c(d, \psi)$.

Proof. Case 1. $r<V^{-1}(\sqrt{t})$.

By Lemmas 3.6 and 3.9 we get

$$
\left|\frac{d}{d r} p_{t}(r)\right| \approx r\left[V^{-1}(\sqrt{t})\right]^{-d-2} \leq c\left[V^{-1}(\sqrt{t})\right]^{-d-1} \approx \frac{p_{t}(r)}{V^{-1}(\sqrt{t})}=\left(\frac{p_{t}(r)}{r} \wedge \frac{p_{t}(r)}{V^{-1}(\sqrt{t})}\right) .
$$

Case 2. $r \geq V^{-1}(\sqrt{t})$.

By Lemmas 3.6 and 3.9 we get

$$
\left|\frac{d}{d r} p_{t}(r)\right| \approx \frac{t}{V^{2}(r) r^{d+1}} \approx \frac{p_{t}(r)}{r}=\left(\frac{p_{t}(r)}{r} \wedge \frac{p_{t}(r)}{V^{-1}(\sqrt{t})}\right) .
$$

Lemma 4.2. For any $t \in(0,1], x, y \in B_{+}(0,1)$ we have

$$
0 \leq p_{t}(x-y)-p_{t}(\hat{x}-y) \leq c|\hat{x}-x|\left(\frac{p_{t}(x-y)}{|x-y|} \wedge \frac{p_{t}(x-y)}{V^{-1}(\sqrt{t})}\right)
$$

where $c=c(d, \psi)$.

Proof. The inequality $p_{t}(x-y)-p_{t}(\hat{x}-y) \geq 0$ is clear because $|\hat{x}-y| \geq|x-y|$.

We also have

$$
\begin{aligned}
p_{t}(x-y)-p_{t}(\hat{x}-y) & =p_{t}(|x-y|)-p_{t}(|\hat{x}-y|) \\
& =(|x-y|-|\hat{x}-y|) D p_{t}(|x-y|+\xi),
\end{aligned}
$$


where $\xi \in(0,|\hat{x}-y|-|x-y|)$ and $D p_{t}(r)=\frac{d}{d r} p_{t}(r)$. By Lemma 4.1 this is bounded from above by

$$
c|\hat{x}-x|\left(\frac{p_{t}(|x-y|+\xi)}{|x-y|+\xi} \wedge \frac{p_{t}(|x-y|+\xi)}{V^{-1}(\sqrt{t})}\right) \leq c|\hat{x}-x|\left(\frac{p_{t}(x-y)}{|x-y|} \wedge \frac{p_{t}(x-y)}{V^{-1}(\sqrt{t})}\right) .
$$

Recall that for $r>0, B=B(0, r), t>0, x, y \in B_{+}$we have

$$
\tilde{p}_{B_{+}}(t, x, y)=p_{B}(t, x, y)-p_{B}(t, \hat{x}, y) .
$$

Lemma 4.3. Let $r \in(0,1], B=B(0, r), x \in B_{+}(0, r / 16), y \in B_{+}$. Then for any $t \in(0,1]$ we have

$$
0 \leq \tilde{p}_{B_{+}}(t, x, y) \leq c|\hat{x}-x|\left(\frac{1}{r} \vee \frac{1}{V^{-1}(\sqrt{t})}\right) p_{B}(t, x, y)
$$

where $c=c(d, \psi)$.

Proof. Let $t_{0}=V^{2}(r) \wedge \frac{t}{2}$ and $t_{1}=t-t_{0}$. By the semigroup property and (14)

$$
\begin{aligned}
\tilde{p}_{B_{+}}(t, x, y) & =\int_{B_{+}} \tilde{p}_{B_{+}}\left(t_{0}, x, w\right) \tilde{p}_{B_{+}}\left(t_{1}, w, y\right) d w \\
& \leq \int_{B_{+}}\left(p_{B}\left(t_{0}, x, w\right)-p_{B}\left(t_{0}, \hat{x}, w\right)\right) p_{B}\left(t_{1}, w, y\right) d w \\
& \leq \int_{B_{+}}\left(p_{t_{0}}(x-w)-p_{t_{0}}(\hat{x}-w)\right) p_{B}\left(t_{1}, w, y\right) d w \\
& \leq c \frac{|\hat{x}-x|}{V^{-1}\left(\sqrt{t_{0}}\right)} \int_{B_{+}} p_{t_{0}}(x-w) p_{B}\left(t_{1}, w, y\right) d w
\end{aligned}
$$

where the last step follows from Lemma 4.2.

By Theorem 2.5, for $w \in B_{+}$, we have

$$
p_{B}\left(t_{1}, y, w\right) \leq c \mathbb{P}^{y}\left(\tau_{B}>\frac{t_{1}}{2}\right) \mathbb{P}^{w}\left(\tau_{B}>\frac{t_{1}}{2}\right) p_{t_{1} \wedge V^{2}(r)}(y-w),
$$

and since $|x| \leq r / 16$ we get

$$
\mathbb{P}^{w}\left(\tau_{B}>\frac{t_{1}}{2}\right) \leq c \mathbb{P}^{x}\left(\tau_{B}>\frac{t_{1}}{2}\right) .
$$

Applying the last two estimates to (26) we obtain

$$
\tilde{p}_{B+}(t, x, y) \leq c \frac{|\hat{x}-x|}{V^{-1}\left(\sqrt{t_{0}}\right)} \mathbb{P}^{x}\left(\tau_{B}>\frac{t_{1}}{2}\right) \mathbb{P}^{y}\left(\tau_{B}>\frac{t_{1}}{2}\right) p_{t_{0}+\left(t_{1} \wedge V^{2}(r)\right)}(x-y) .
$$

Note that $t_{0}+\left(t_{1} \wedge V^{2}(r)\right) \approx t \wedge V^{2}(r)$, which implies, by Lemma 3.6, that

$$
p_{t_{0}+\left(t_{1} \wedge V^{2}(r)\right)}(x-y) \approx p_{t \wedge V^{2}(r)}(x-y) .
$$

Moreover,

$$
\mathbb{P}^{x}\left(\tau_{B}>\frac{t_{1}}{2}\right) \approx \mathbb{P}^{x}\left(\tau_{B}>\frac{t}{2}\right), \quad \mathbb{P}^{y}\left(\tau_{B}>\frac{t_{1}}{2}\right) \approx \mathbb{P}^{y}\left(\tau_{B}>\frac{t}{2}\right),
$$

which follows from Theorem 2.5 and (17). 
Finally, we infer that

$$
\tilde{p}_{B_{+}}(t, x, y) \leq c \frac{|\hat{x}-x|}{V^{-1}\left(\sqrt{t_{0}}\right)} p_{t \wedge V^{2}(r)}(x-y) \mathbb{P}^{x}\left(\tau_{B}>\frac{t}{2}\right) \mathbb{P}^{y}\left(\tau_{B}>\frac{t}{2}\right) .
$$

Note that $V^{-1}\left(\sqrt{t_{0}}\right)=r \wedge V^{-1}(\sqrt{t / 2})$. By scaling properties of $V^{-1}$ from Lemma 2.1 we get

$$
\frac{1}{V^{-1}\left(\sqrt{t_{0}}\right)}=\frac{1}{r} \vee \frac{1}{V^{-1}(\sqrt{t / 2})} \leq c\left(\frac{1}{r} \vee \frac{1}{V^{-1}(\sqrt{t})}\right) \text {. }
$$

Observing, again by Theorem 2.5, that we have

$$
p_{B}(t, x, y) \approx p_{t \wedge V^{2}(r)}(x-y) P^{x}\left(\tau_{B}>\frac{t}{2}\right) P^{y}\left(\tau_{B}>\frac{t}{2}\right)
$$

we complete the proof.

Lemma 4.4. Let $r \in(0,1], B=B(0, r), x \in B_{+}(0, r / 16), x=|x| e_{1}, y \in$ $B_{+}(0, r / 4)$. Then for every $t \in(0,1]$ we have

$$
\tilde{p}_{B_{+}}(t, x, y) \leq c \frac{|x-\hat{x}|}{|y|} p_{B}(t, x, y)
$$

where $c=c(d, \psi)$.

Proof. Case 1. $|y| \leq 4|x|$.

We have

$$
\tilde{p}_{B_{+}}(t, x, y) \leq p_{B}(t, x, y) \leq \frac{2|x-\hat{x}|}{|y|} p_{B}(t, x, y) .
$$

Case 2. $|y|>4|x|, t \leq V^{2}(|x-y|)$.

Note that $|y-x| \approx|y|$. By (14), Lemma 4.2 and (18) we get (27).

Case 3. $|y|>4|x|, t>V^{2}(|x-y|)$.

By Lemma 4.3 we arrive at

$$
\tilde{p}_{B_{+}}(t, x, y) \leq c|\hat{x}-x|\left(\frac{1}{r} \vee \frac{1}{V^{-1}\left(\sqrt{V^{2}(|x-y|)}\right)}\right) p_{B}(t, x, y) \leq c \frac{|x-\hat{x}|}{|y|} p_{B}(t, x, y) \text {. }
$$

Lemma 4.5. Let $r \in(0,1], t \in(0,1], B=B(0, r), x \in B_{+}(0, r / 16), y \in B_{+} \backslash$ $B_{+}(0, r / 4)$. Then

$$
\tilde{p}_{B_{+}}(t, x, y) \leq c \frac{|\hat{x}-x|}{r} p_{B}(t, x, y)
$$

where $c=c(d, \psi)$.

Proof. Note that for $t \in\left(V^{2}(r) \wedge 1,1\right]$ we have

$$
\frac{1}{V^{-1}(\sqrt{t})} \leq \frac{1}{V^{-1}\left(\sqrt{V^{2}(r)}\right)}=\frac{1}{r}
$$

Hence the assertion of the lemma for $t \in\left(V^{2}(r) \wedge 1,1\right]$ follows from Lemma 4.3. So we may assume that $t \in\left(0, V^{2}(r) \wedge 1\right]$.

Observe that $|x-y| \geq 3 r / 16$. If $y \in B_{+}(0,3 r / 4) \backslash B_{+}(0, r / 4)$ then by (14), Lemma 4.2 and (18) we get

$$
\tilde{p}_{B_{+}}(t, x, y) \leq c \frac{|\hat{x}-x|}{r} p_{t}(x-y) \leq c \frac{|\hat{x}-x|}{r} p_{B}(t, x, y),
$$


so we may assume that $y \in B_{+} \backslash B_{+}(0,3 r / 4)$.

Let $D^{*}=B(0, r) \backslash \overline{B(0, r / 2)}, D_{0}=\left\{z \in \mathbb{R}_{+}^{d}: r / 4 \leq|z| \leq r / 2\right\}, D_{1}=B_{+}(0,4|x|)$ and $D_{2}=B_{+}(0, r / 4)$. By standard arguments (the strong Markov property of $\tilde{X}$ and $(16))$ we have

$$
\tilde{p}_{B_{+}}(t, x, y)=\int_{D_{+}^{*}} \int_{0}^{t} \tilde{p}_{D_{+}^{*}}(s, y, w) \int_{B_{+} \backslash D_{+}^{*}} \tilde{\nu}(w, z) \tilde{p}_{B_{+}}(t-s, z, x) d z d s d w .
$$

Splitting the integration we obtain

$$
\begin{aligned}
\tilde{p}_{B_{+}}(t, x, y) & =\int_{D_{+}^{*}} \int_{0}^{t} \tilde{p}_{D_{+}^{*}}(s, y, w) \int_{D_{0}} \tilde{\nu}(w, z) \tilde{p}_{B_{+}}(t-s, z, x) d z d s d w \\
& +\int_{D_{+}^{*}} \int_{0}^{t} \tilde{p}_{D_{+}^{*}}(s, y, w) \int_{D_{1}} \tilde{\nu}(w, z) \tilde{p}_{B_{+}}(t-s, z, x) d z d s d w \\
& +\int_{D_{+}^{*}} \int_{0}^{t} \tilde{p}_{D_{+}^{*}}(s, y, w) \int_{D_{2} \backslash D_{1}} \tilde{\nu}(w, z) \tilde{p}_{B_{+}}(t-s, z, x) d z d s d w \\
& =\mathrm{I}+\mathrm{II}+\mathrm{III} .
\end{aligned}
$$

First we estimate I. By (14) and Lemma 4.2, for $r / 4 \leq|z| \leq r / 2, s \in(0, t)$, we have

$$
\tilde{p}_{B_{+}}(t-s, z, x) \leq c|x-\hat{x}| \frac{p_{t-s}(x-z)}{|x-z|} .
$$

Since $|x-z| \approx|x-y| \approx r$, by Lemma 3.6 and the subadditivity of $V$, this is bounded from above by

$$
c|x-\hat{x}| \frac{p_{t}(x-y)}{r} .
$$

Hence, using the estimate $P^{y}\left(r / 4 \leq\left|X\left(\tau_{D^{*}}\right)\right| \leq r / 2\right) \leq c \frac{V\left(\delta_{B}(y)\right)}{V(r)}[20$, see Lemma 5.7], we obtain

$$
\begin{aligned}
\mathrm{I} & \leq c \frac{|x-\hat{x}|}{r} p_{t}(x-y) \int_{D^{*}} \int_{0}^{t} p_{D^{*}}(s, y, w) \int_{D_{0}} \nu(w-z) d z d s d w \\
& \leq c \frac{|x-\hat{x}|}{r} p_{t}(x-y) P^{y}\left(r / 4 \leq\left|X\left(\tau_{D^{*}}\right)\right| \leq r / 2\right) \\
& \leq c \frac{|x-\hat{x}|}{r} p_{t}(x-y) \frac{V\left(\delta_{B}(y)\right)}{V(r)} \\
& \leq c \frac{|x-\hat{x}|}{r} p_{B}(t, x, y)
\end{aligned}
$$

where the last step follows from Theorem 2.5 and (17).

By Lemmas 2.3, 3.4 and the subadditivity of $V$ we obtain

$$
\tilde{\nu}(w, z) \leq c|z| \frac{\nu(w-z)}{r} \approx|z| \frac{\nu(x-y)}{r},
$$

for $w \in D_{+}^{*}$ and $z \in D_{2}$. In particular, $\tilde{\nu}(w, z) \leq \frac{c}{r}|x-\hat{x}| \nu(w-z)$, for $w \in D_{+}^{*}$ and $z \in D_{1}$. Hence, we get

$$
\begin{aligned}
\mathrm{II} & \leq c \frac{|x-\hat{x}|}{r} \int_{D^{*}} \int_{0}^{t} p_{D^{*}}(s, y, w) \int_{D_{1}} \nu(w-z) p_{B}(t-s, z, x) d z d s d w \\
& \leq c \frac{|x-\hat{x}|}{r} p_{B}(t, y, x) .
\end{aligned}
$$


Next, using (14) and Lemma 4.2, for $s \in(0, t), z \in D_{2} \backslash D_{1}$ we get

$$
\tilde{p}_{B_{+}}(t-s, z, x) \leq c \frac{|x-\hat{x}|}{|x-z|} p_{t-s}(x-z) \leq c \frac{|x-\hat{x}|}{|z|} p_{t-s}(x-z) .
$$

Moreover, since $0<t \leq V^{2}(r)$, by (18), for $s \in(0, t), z \in D_{2} \backslash D_{1}$ we get

$$
p_{t-s}(x-z) \approx p_{B}(t-s, z, x) .
$$

Combining (28), (29) and (30) we obtain

$$
\begin{aligned}
\mathrm{III} & \leq c \frac{|x-\hat{x}|}{r} \int_{D_{+}^{*}} \int_{0}^{t} \tilde{p}_{D_{+}^{*}}(s, y, w) \int_{D_{2} \backslash D_{1}} \nu(w-z) p_{B}(t-s, z, x) d z d s d w \\
& \leq c \frac{|x-\hat{x}|}{r} \int_{D^{*}} \int_{0}^{t} p_{D^{*}}(s, y, w) \int_{D_{2} \backslash D_{1}} \nu(w-z) p_{B}(t-s, z, x) d z d s d w \\
& \leq c \frac{|x-\hat{x}|}{r} p_{B}(t, y, x) .
\end{aligned}
$$

The proof is completed.

Proposition 4.6. Let $D$ be an open set, $0 \in D$ and $r=\delta_{D}(0) \wedge 1$. Let $x=$ $|x| e_{1},|x|<r / 16$ and $y \in D$. For any $t \in(0,1]$ we have

$$
\left|p_{D}(t, x, y)-p_{D}(t, \hat{x}, y)\right| \leq c|\hat{x}-x|\left[\frac{1}{r} \vee \frac{1}{V^{-1}(\sqrt{t})}\right] p_{D}(t, x, y),
$$

where $c=c(d, \psi)$.

Proof. We set $B=B(0, r)$ and we put

$$
k_{D}(t, x, y)=\int_{B} \int_{0}^{t} p_{B}(s, x, w) \int_{D \backslash B} \nu(w-z) p_{D}(t-s, z, y) d z d s d w .
$$

By standard arguments (the strong Markov property and (12))

$$
p_{D}(t, x, y)=p_{B}(t, x, y)+k_{D}(t, x, y) .
$$

Lemma 4.3 yields the estimate

$$
\left|p_{B}(t, x, y)-p_{B}(t, \hat{x}, y)\right| \leq c|\hat{x}-x|\left[\frac{1}{r} \vee \frac{1}{V^{-1}(\sqrt{t})}\right] p_{D}(t, x, y) .
$$

Next, we estimate $\left|k_{D}(t, x, y)-k_{D}(t, \hat{x}, y)\right|$. For $s \in(0, t), w \in B$, let

$$
g_{s}(w)=\int_{D \backslash B} \nu(w-z) p_{D}(t-s, z, y) d z .
$$

Note that for $w \in B_{+}(0, r / 4)$ and $z \in D \backslash B$ we have, due to Lemma 2.3 .

$$
|\nu(w-z)-\nu(\hat{w}-z)| \leq c \frac{|w|}{r} \nu(w-z) .
$$

Hence, for $s \in(0, t), w \in B_{+}(0, r / 4)$,

$$
\begin{aligned}
\left|g_{s}(w)-g_{s}(\hat{w})\right| & \leq \int_{D \backslash B}|\nu(w-z)-\nu(\hat{w}-z)| p_{D}(t-s, z, y) d z \\
& \leq c \frac{|w|}{r} \int_{D \backslash B} \nu(w-z) p_{D}(t-s, z, y) d z \\
& =c \frac{|w|}{r} g_{s}(w) .
\end{aligned}
$$


By simple manipulations we obtain

$$
\begin{aligned}
k_{D}(t, x, y)-k_{D}(t, \hat{x}, y) & =\int_{B_{+}} \int_{0}^{t}\left(p_{B}(s, x, w)-p_{B}(s, \hat{x}, w)\right)\left(g_{s}(w)-g_{s}(\hat{w})\right) d s d w \\
& =\int_{B_{+}(0, r / 4)} \int_{0}^{t}+\int_{B_{+} \backslash B_{+}(0, r / 4)} \int_{0}^{t} \\
& =\mathrm{I}+\mathrm{II} .
\end{aligned}
$$

By Lemma 4.4 and (33) we get

$$
\begin{aligned}
|\mathrm{I}| & \leq c \int_{B_{+}(0, r / 4)} \int_{0}^{t}\left(p_{B}(s, x, w)-p_{B}(s, \hat{x}, w)\right) \frac{|w|}{r} g_{s}(w) d s d w \\
& \leq c \frac{|x-\hat{x}|}{r} \int_{B(0, r / 4)} \int_{0}^{t} p_{B}(s, x, w) g_{s}(w) d s d w \\
& \leq c \frac{|x-\hat{x}|}{r} k_{D}(t, x, y) .
\end{aligned}
$$

Note that by Theorem 2.5, the subadditivity of $V$ and Lemma 3.6, for $s \in(0, t)$ and $w \in B \backslash B(0, r / 4)$, we have

$$
p_{B}(s, x, w) \approx p_{B}(s, 0, w) .
$$

Using this and Lemma 4.5 we get

$$
\begin{aligned}
|\mathrm{II}| & \leq c \frac{|x-\hat{x}|}{r} \int_{B} \int_{0}^{t} p_{B}(s, 0, w)\left(g_{s}(w)+g_{s}(\hat{w})\right) d s d w \\
& =2 c \frac{|x-\hat{x}|}{r} \int_{B} \int_{0}^{t} p_{B}(s, 0, w) g_{s}(w) d s d w \\
& \approx \frac{|x-\hat{x}|}{r} \int_{B} \int_{0}^{t} p_{B}(s, x, w) g_{s}(w) d s d w \\
& =\frac{|x-\hat{x}|}{r} k_{D}(t, x, y) .
\end{aligned}
$$

Hence,

$$
\left|k_{D}(t, x, y)-k_{D}(t, \hat{x}, y)\right| \leq c \frac{|x-\hat{x}|}{r} k_{D}(t, x, y)
$$

which combined with the estimate (32) completes the proof.

Lemma 4.7. Let $D \subset \mathbb{R}^{d}$ be an open set. $\nabla_{x} p_{D}(t, x, y)$ is well defined for any $t>0$, $x, y \in D$.

Proof. Recall that

$$
p_{D}(t, x, y)=p_{t}(x-y)-E^{y}\left(p_{t-\tau_{D}}\left(X\left(\tau_{D}\right)-x\right), \tau_{D}<t\right), \quad t>0, x, y \in D .
$$

Since $\psi$ satisfies the Hartman-Wintner condition it is well known that for each $t>0$ the function $x \rightarrow p_{t}(x)$ has derivatives of all orders on $\mathbb{R}^{d}$ [18, Lemma 3.1]. By Theorem 3.7 and Lemma 3.2 for any $s \in(0, t), x \in D, \nabla_{x} p_{s}\left(x-X\left(\tau_{D}\right)\right)$ is well defined and

where $c=c(d, \psi)$.

$$
\left|\nabla_{x} p_{s}\left(x-X\left(\tau_{D}\right)\right)\right| \leq \frac{c t}{V^{2}\left(\delta_{D}(x)\right) \delta_{D}^{d+1}(x)}
$$


So by the bounded convergence theorem $\nabla_{x} E^{y}\left(p_{t-\tau_{D}}\left(X\left(\tau_{D}\right)-x\right), \tau_{D}<t\right)$ is well defined for any $t>0, x, y \in D$.

proof of Theorem 1.1. The existence of $\nabla_{x} p_{D}(t, x, y)$ follows from Lemma 4.7.

Denote $D_{i} p_{D}(t, x, y)=\frac{\partial}{\partial x_{i}} p_{D}(t, x, y)$. Choose $z, y \in D$ and put $r=\delta_{D}(z) \wedge 1$. We will estimate $D_{1} p_{D}(t, z, y)$. Estimates for $D_{i} p_{D}(t, z, y), i \neq 1$ may be obtained in the same way. We may assume that $z=0$. Choose $\varepsilon \in(0, r / 16)$. Putting $x=h e_{1}$, $(h \in(0, \varepsilon))$ in (31) we obtain

$$
\sup _{0<h<\varepsilon}\left|\frac{p_{D}\left(t, h e_{1}, y\right)-p_{D}\left(t,-h e_{1}, y\right)}{2 h}\right| \leq c\left[\frac{1}{r} \vee \frac{1}{V^{-1}(\sqrt{t})}\right] \sup _{0<h<\varepsilon} p_{D}\left(t, h e_{1}, y\right),
$$

which implies

$$
\left|D_{1} p_{D}(t, z, y)\right| \leq c\left[\frac{1}{r} \vee \frac{1}{V^{-1}(\sqrt{t})}\right] p_{D}(t, z, y) .
$$

Finally using (7) we obtain (1).

\section{ExAmples}

Example 5.1. Let $X$ be the isotropic $\alpha$-stable process in $\mathbb{R}^{d}, \alpha \in(0,2), d \in \mathbb{N}$. We have $\psi(x)=|x|^{\alpha}$ and $\nu(x)=C_{d, \alpha}|x|^{-d-\alpha}$, where $C_{d, \alpha}=\frac{2^{\alpha} \Gamma((d+\alpha) / 2)}{\pi^{d / 2}|\Gamma(-\alpha / 2)|}$. It is clear that $X$ satisfies assumptions of Theorem 1.1. Hence, for any open, nonempty set $D \subset \mathbb{R}^{d}$ we have

$$
\left|\nabla_{x} p_{D}(t, x, y)\right| \leq \frac{c}{\delta_{D}(x) \wedge t^{1 / \alpha}} p_{D}(t, x, y), \quad x, y \in D, t \in(0,1],
$$

where $c=c(d, \alpha)$.

Example 5.2. Let $X$ be the the relativistic process in $\mathbb{R}^{d}$, (see e.g. [5], [28]). We have $\psi(x)=\sqrt{|x|^{2}+m^{2}}-m, m>0$,

$$
\nu(x)=2^{\frac{1-d}{2}} \pi^{\frac{-d-1}{2}} m^{\frac{d+1}{2}}|x|^{\frac{-d-1}{2}} K_{\frac{d+1}{2}}(m|x|),
$$

where $K_{s}(r), s \in \mathbb{R}$, is the modified Bessel function of the second kind with index $s$ (called also Macdonald function), given by

$$
K_{s}(r)=2^{-1-s} r^{s} \int_{0}^{\infty} e^{-u} e^{-r^{2} /(4 u)} u^{-1-s} d u, \quad r>0 .
$$

The generator of this process $m-\sqrt{m^{2}-\Delta}$ is called the relativistic Hamiltonian and it is used in some models of mathematical physics (see e.g. [23]). One can check that $X$ satisfies assumptions of Theorem 1.1. Hence, for any open, nonempty set $D \subset \mathbb{R}^{d}$ we have

$$
\left|\nabla_{x} p_{D}(t, x, y)\right| \leq \frac{c}{\delta_{D}(x) \wedge t} p_{D}(t, x, y), \quad x, y \in D, t \in(0,1],
$$

where $c=c(d, m)$.

Example 5.3. Let $X_{t}=B_{S_{t}}$ where $B$ is the Brownian motion in $\mathbb{R}^{d}$ (with a generator $\Delta$ ) and $S$ is an independent subordinator with the Laplace exponent $\phi$. We assume that the Levy measure of the subordinator $S$ is infinite, $\phi$ is a complete Bernstein function and it satisfies

$$
c_{1} \lambda^{\alpha / 2} \ell(\lambda) \leq \phi(\lambda) \leq c_{2} \lambda^{\alpha / 2} \ell(\lambda), \quad \lambda \geq 1,
$$


where $0<\alpha<2$, $\ell$ varies slowly at infinity, i.e. $\forall x>0 \lim _{\lambda \rightarrow \infty} \frac{\ell(\lambda x)}{\ell(\lambda)}=1$. (Clearly processes from Examples 5.1, 5.2 satisfies these assumptions).

We have $\psi(x)=\phi\left(|x|^{2}\right)$. By (36) $\psi$ satisfies $\operatorname{WLSC}\left(\underline{\alpha}, \theta_{0}, \underline{C}\right)$ and $\operatorname{WUSC}\left(\bar{\alpha}, \theta_{0}, \bar{C}\right)$ for some $\underline{\alpha}>0, \bar{\alpha} \in(0,2), \theta_{0} \geq 0$, and $\underline{C}, \bar{C}>0$. The assumptions concerning the Lévy measure in Theorem 1.1 are satisfied by [20, Proposition 1.3 and the proof of Example 7.1].

Hence, for any open, nonempty set $D \subset \mathbb{R}^{d}$ we have

$$
\left|\nabla_{x} p_{D}(t, x, y)\right| \leq c\left[\frac{1}{\delta_{D}(x) \wedge 1} \vee \sqrt{\phi^{-1}(1 / t)}\right] p_{D}(t, x, y), \quad x, y \in D, t \in(0,1],
$$

where $c=c(d, \phi)$.

The process in the next example is not a subordinate Brownian motion cf. [20, Example 7.4].

Example 5.4. Let $\left\{X_{t}\right\}$ be the pure-jump isotropic Lévy process in $\mathbb{R}^{d}$ with the Lévy measure $\nu(d x)=\nu(|x|) d x$ given by the formula

$$
\nu(r)=\left\{\begin{array}{lll}
\mathcal{A}_{d, \alpha} r^{-d-\alpha} & \text { for } & r \in(0,1] \\
c_{1} e^{-c_{2} r} & \text { for } & r \in(1, \infty)
\end{array}\right.
$$

where $\mathcal{A}_{d, \alpha} r^{-d-\alpha}$ is the Lévy density for the isotropic $\alpha$-stable process in $\mathbb{R}^{d}, \alpha \in$ $(0,2), d \in \mathbb{N}$ and $c_{1}=\mathcal{A}_{d, \alpha} e^{d+\alpha}>0, c_{2}=d+\alpha>0$ are chosen so that $\nu(r) \in$ $C^{1}(0, \infty)$.

Note that $\psi(x)=\int_{\mathbb{R}^{d}}(1-\cos \langle x, y\rangle) \nu(d y)$ behaves for $|x| \geq 1$ like the characteristic exponent for the isotropic $\alpha$-stable process so it satisfies

$$
c_{1}|x|^{\alpha} \leq \psi(x) \leq c_{2}|x|^{\alpha}, \quad|x| \geq 1,
$$

where $c_{1}=c_{1}(d, \alpha), c_{2}=c_{2}(d, \alpha)$. Hence, $\left.\psi \in \operatorname{WLSC}(\alpha, 1, \underline{C})\right) \cap \operatorname{WUSC}(\alpha, 1, \bar{C})$ for some $\underline{C}, \bar{C}>0$.

The assumptions concerning the Lévy measure in Theorem 1.1 are easy to check.

Note that we have $\psi^{-}(x) \approx|x|^{1 / \alpha}$ for $|x| \geq 1$, where the comparability constant depends only on $d$ and $\alpha$. Hence, for any open, nonempty set $D \subset \mathbb{R}^{d}$ we have

$$
\left|\nabla_{x} p_{D}(t, x, y)\right| \leq \frac{c}{\delta_{D}(x) \wedge t^{1 / \alpha}} p_{D}(t, x, y), \quad x, y \in D, t \in(0,1]
$$

where $c=c(d, \alpha)$.

\section{Appendix}

The section is devoted to the proof of Theorem 2.5. A similar result for smooth bounded domains was proved in [3, Theorem 4.5], but the dependence of constants therein seems to be unclear and one can not infer uniform estimates of the Dirichlet heat kernels as in Theorem 2.5. We follow the arguments from the proof [3, Theorem 4.5], but we pay more attention to the behaviour of the constants. To make the exposition self-contained we need to introduce some notation and to cite several results obtained in [1, 3]. For $R>0$ we denote $B_{R}=B(0, R)$. Let $\underline{\alpha}>0$. For $R>0$ 
we introduce the following quantities:

$$
\begin{aligned}
\underline{C}_{R} & =\inf _{y \geq x \geq \frac{1}{R}} \frac{\psi(y)}{\psi(x)}\left(\frac{x}{y}\right)^{\underline{\alpha}} ; \\
\tilde{C}_{R} & =\inf _{0<t \leq V^{2}(R),|x| \leq R} \frac{p_{t}(x)}{p_{t / 2}(0) \wedge \frac{t}{V^{2}(|x|)|x|^{d}}} ; \\
C_{R} & =1 \wedge \inf _{0<t \leq V^{2}(|x|),|x| \leq R} \frac{p_{t}(x)}{t} V^{2}(|x|)|x|^{d} ; \\
C_{R}^{*} & =\inf _{|x| \leq R} \nu(x) V^{2}(|x|)|x|^{d} ; \\
\mathcal{J}_{R} & =\inf _{0<\rho \leq R / 2} \nu\left(B_{R} \backslash B_{\rho}\right) V^{2}(\rho) .
\end{aligned}
$$

Remark 6.1. At first we observe that if there exists $R>0$ such that $\underline{C}_{R}>0$ then we get $\psi \in \mathrm{WLSC}\left(\underline{\alpha}, \frac{1}{R}, \underline{C_{R}}\right)$. Consequently, due to [2, Lemma 12], $\underline{C}_{R}>0$ for any $R>0$. On the other hand, if there exists $R>0$ and $\underline{C}>0$ such that $\psi \in \operatorname{WLSC}\left(\underline{\alpha}, \frac{1}{R}, \underline{C}\right)$ then we get $\underline{C}_{R} \geq \underline{C}$.

Remark 6.2. If $R>0, \psi \in \operatorname{WLSC}\left(\underline{\alpha}, \theta_{0}, \underline{C}\right) \cap \operatorname{WUSC}\left(\bar{\alpha}, \theta_{0}, \bar{C}\right)$, for some $\underline{\alpha}>0$, $\bar{\alpha} \in(0,2), \theta_{0} \geq 0, \underline{C}, \bar{C}>0$ and the Lévy measure has strictly positive density then the constants $\tilde{C}_{R}, C_{R}, C_{R}^{*}, \mathcal{J}_{R}$ are strictly positive. Indeed, by Lemma 3.4 we get $C_{R}^{*}>0$. By Lemma 3.6 we get $\tilde{C}_{R} \wedge C_{R}>0$. Moreover, by elementary calculations $\mathcal{J}_{R} \geq c(d) C_{R}^{*}$ and $C_{R} \geq c(d) \tilde{C}_{R}$.

Lemma 6.3. [3, Lemma 1.6] If $\psi \in \operatorname{WLSC}\left(\underline{\alpha}, \theta_{0}, \underline{C}\right), r>0$ and $0<t \leq r V^{2}\left(1 / \theta_{0}\right)$, then

$$
c_{2} e^{-c_{1} r}\left[V^{-1}(\sqrt{t / r})\right]^{-d} \leq p_{t}(0) \leq c_{3}\left(1+(\underline{C} r)^{-1-d / \underline{\alpha}}\right)\left[V^{-1}(\sqrt{t / r})\right]^{-d},
$$

where $c_{1}$ is an absolute constant, $c_{2}=c_{2}(d)$ and $c_{3}=c_{3}(d, \underline{\alpha})$.

Corollary 6.4. Let $R>0$ and $0<r \leq 1$. Under the assumptions of the previous lemma there are $c_{1}=c_{1}(d)$ and $c_{2}=c_{2}(d, \underline{\alpha})$ such that

$$
c_{1} \frac{1}{R^{d}} \leq p_{r V^{2}(R)}(0) \leq c_{2} \frac{1}{\left(\underline{C}_{R} r\right)^{1+d / \underline{\alpha}}} \frac{1}{R^{d}} .
$$

Proof. If $\psi \in \operatorname{WLSC}\left(\underline{\alpha}, \theta_{0}, \underline{C}\right)$ then $\underline{C}_{R}>0$ and $\psi \in \operatorname{WLSC}\left(\underline{\alpha}, 1 / R, \underline{C}_{R}\right)$. Therefore, the conclusion follows from Lemma 6.3 with $\theta_{0}=1 / R, \underline{C}=\underline{C}_{R} \leq 1$.

Lemma 6.5. [1, Proposition 6.1] Let the condition $(\boldsymbol{H})$ hold. There are $c_{1}=c_{1}(d)<$ 1 and $c_{2}=c_{2}(d)$ such that for $R>0$,

$$
\mathbb{P}^{x}\left(\tau_{B_{R}}>t\right) \geq c_{2} \frac{\mathcal{J}_{R}}{H_{R}}\left(\frac{V\left(\delta_{B_{R}}(x)\right)}{\sqrt{t}} \wedge 1\right), \quad 0<t \leq c_{1} V^{2}(R), \quad x \in \mathbb{R}^{d} .
$$

Lemma 6.6. [3], Corollary 2.8] Let $D$ be open and convex. If $\psi \in \operatorname{WLSC}\left(\underline{\alpha}, \theta_{0}, \underline{C}\right)$, $t>0,|x-y|<1 / \theta_{0}$ and $x, y \in D$, then there is a constant $C=C(d, \underline{\alpha})$ such that for all $t>0$,

$$
\begin{aligned}
p_{D}(t, x, y) \leq & \frac{C}{\underline{C}^{2(1+d) / \underline{\alpha}+1}}\left(\frac{V\left(\delta_{D}(x)\right)}{\sqrt{t}} \wedge 1\right)\left(\frac{V\left(\delta_{D}(y)\right)}{\sqrt{t}} \wedge 1\right) \\
& \times\left(p_{t / 2}(0) \wedge \frac{t}{V^{2}(|x-y|)|x-y|^{d}}\right) .
\end{aligned}
$$


Lemma 6.7. [3, Lemma 4.2] Let $D$ be a bounded open set and $t_{0}>0$. For $t \geq t_{0}$ and $x, y \in D$,

$$
p_{D}(t, x, y) \leq|D|\left(p_{t_{0} / 4}(0)\right)^{2} \mathbb{P}^{x}\left(\tau_{D}>\frac{t_{0}}{4}\right) \mathbb{P}^{y}\left(\tau_{D}>\frac{t_{0}}{4}\right) e^{\lambda_{1} t_{0}} e^{-\lambda_{1} t}
$$

where $\lambda_{1}=\lambda_{1}^{D}$ and $|D|$ is the volume of $D$.

To get the uniform lower bound of the heat kernel we use the following result, which is a direct consequence of [3, Theorem 3.3].

Lemma 6.8. Let $R>0$. Assume that $\psi \in \operatorname{WLSC}\left(\underline{\alpha}, \theta_{0}, \underline{C}\right) \cap \operatorname{WUSC}\left(\bar{\alpha}, \theta_{0}, \bar{C}\right)$, for some $\underline{\alpha}>0, \bar{\alpha} \in(0,2), \theta_{0} \geq 0, \underline{C}, \bar{C}>0$ and the Lévy measure has strictly positive density. Then there exist $c=c(d)<1, c_{1}=c_{1}(d, \underline{\alpha})$ such that

$$
\begin{aligned}
p_{B_{R}}(t, x, y) \geq & \frac{c_{1}\left(\underline{C}_{R}\right)^{1+d / \underline{\alpha}}\left(C_{R}\right)^{9+d / \underline{\alpha}}}{H_{R}^{2}}\left(\frac{V\left(\delta_{B_{R}}(x)\right)}{\sqrt{t}} \wedge 1\right)\left(\frac{V\left(\delta_{B_{R}}(y)\right)}{\sqrt{t}} \wedge 1\right) \\
& \times\left(p_{t / 2}(0) \wedge[t \nu(2|x-y|)]\right),
\end{aligned}
$$

provided $0<t \leq c V^{2}(R) C_{R}$ and $x, y \in B_{R}$.

To deal with the lower bound for large $t$ we use the following result.

Lemma 6.9. [3, Lemma 4.3] Let $D$ be a bounded open set. If $t_{0}>0$ and $c_{*}>0$ are such that

$$
p_{D}\left(\frac{t_{0}}{2}, x, y\right) \geq c_{*} \mathbb{P}^{x}\left(\tau_{D}>\frac{t_{0}}{2}\right) \mathbb{P}^{y}\left(\tau_{D}>\frac{t_{0}}{2}\right), \quad x, y \in D
$$

then for $t \geq t_{0}$ and $x, y \in D$,

$$
p_{D}(t, x, y) \geq\left(\frac{c_{*}}{\sqrt{|D|} p_{t_{0} / 2}(0)}\right)^{2} e^{-\lambda_{1} t_{0}} \mathbb{P}^{x}\left(\tau_{D}>\frac{t_{0}}{2}\right) \mathbb{P}^{y}\left(\tau_{D}>\frac{t_{0}}{2}\right) e^{-\lambda_{1} t} .
$$

Now, we are in a position to prove Theorem 2.5 .

Proof of Theorem 2.5. Fix $R>0$. In the whole proof we understand that all inequalities hold for all $x, y \in B_{R}$. Observe that $\psi \in \mathrm{WLSC}\left(\underline{\alpha}, \frac{1}{2 R}, \underline{C}_{2 R}\right)$. Hence, using Lemma 6.6 for $D=B_{R}$, we find a constant $c_{1}=c_{1}(d, \underline{\alpha})$ such that for any $t>0$,

$$
\begin{aligned}
p_{B_{R}}(t, x, y) \leq & \frac{c_{1}}{\underline{C}_{2 R}^{2(1+d) / \underline{\alpha}+1}}\left(\frac{V\left(\delta_{B_{R}}(x)\right)}{\sqrt{t}} \wedge 1\right)\left(\frac{V\left(\delta_{B_{R}}(y)\right)}{\sqrt{t}} \wedge 1\right) \\
& \times\left(p_{t / 2}(0) \wedge \frac{t}{V^{2}(|x-y|)|x-y|^{d}}\right) .
\end{aligned}
$$

Let $t_{0}=V^{2}(R), \lambda_{1}=\lambda_{1}^{B(0, R)}$. By Lemma 2.4, $\lambda_{1} t_{0} \leq c(d)$. Moreover, for $t \leq t_{0}$ we have the estimate

$$
\left(p_{t / 2}(0) \wedge \frac{t}{V^{2}(|x-y|)|x-y|^{d}}\right) \leq \frac{1}{\tilde{C}_{2 R}} p_{t}(x-y) .
$$

Consequently, applying (40), for $t \leq t_{0}$ we obtain

$$
\begin{aligned}
p_{B_{R}}(t, x, y) \leq & \frac{c_{2}}{\tilde{C}_{2 R} \underline{C}_{2 R}^{2(1+d) / \underline{\alpha}+1}}\left(\frac{V\left(\delta_{B_{R}}(x)\right)}{\sqrt{t}} \wedge 1\right)\left(\frac{V\left(\delta_{B_{R}}(y)\right)}{\sqrt{t}} \wedge 1\right) \\
& \times p_{t}(x-y) e^{-\lambda_{1} t}
\end{aligned}
$$


with $c_{2}=c_{2}(d, \underline{\alpha})$.

Next, we deal with $t \geq t_{0}$. By Corollary 6.4 with $r=1 / 4, p_{t_{0} / 4}(0) \leq \frac{c_{3}}{\underline{C}_{R}^{1+d / \alpha}} \frac{1}{R^{d}}$ where $c_{3}=c_{3}(d, \underline{\alpha})$. Moreover, subadditivity of $V$ yields $\frac{1}{R^{d}} \leq \frac{2^{d+2}}{C_{2 R}} p_{V^{2}(R)}(2 R) \leq$ $\frac{2^{d+2}}{C_{2 R}} p_{V^{2}(R)}(x-y)$. Applying Lemma 6.7 for $D=B_{R}$ with $t_{0}=V^{2}(R)$, we obtain for $t \geq t_{0}$

$$
p_{B_{R}}(t, x, y) \leq \frac{c_{4}}{C_{2 R} \underline{C}_{R}^{2(1+d / \underline{\alpha})}} p_{V^{2}(R)}(x-y) \mathbb{P}^{x}\left(\tau_{D}>\frac{t_{0}}{4}\right) \mathbb{P}^{y}\left(\tau_{D}>\frac{t_{0}}{4}\right) e^{-\lambda_{1} t}
$$

where $c_{4}=c_{4}(d, \underline{\alpha})$. We also have (see [22, Theorem 3.1])

$$
\mathbb{P}^{x}\left(\tau_{B_{R}}>t\right) \leq c_{5}\left(\frac{V\left(\delta_{B_{R}}(x)\right)}{\sqrt{t}} \wedge 1\right)
$$

for an absolute constant $c_{5}$. Hence, for $t \geq t_{0}=V^{2}(R)$, we arrive at

$p_{B_{R}}(t, x, y) \leq \frac{c_{6}}{C_{2 R} \underline{C}_{R}^{2(1+d / \underline{\alpha})}} p_{V^{2}(R)}(x-y)\left(\frac{V\left(\delta_{B_{R}}(x)\right)}{\sqrt{t} \wedge V(R)} \wedge 1\right)\left(\frac{V\left(\delta_{B_{R}}(y)\right)}{\sqrt{t} \wedge V(R)} \wedge 1\right) e^{-\lambda_{1} t}$

with $c_{6}=c_{6}(d, \underline{\alpha})$.

Therefore, by (41) and (43)), we can find $\mathcal{A}_{R}=\frac{c_{6}}{C_{2 R} \underline{C}_{R}^{2(1+d / \underline{\alpha})}}+\frac{c_{2}}{\tilde{C}_{2 R} \underline{C}_{2 R}^{2(1+d) / \underline{\alpha}+1}}$ such that for all $t>0$

$$
p_{B_{R}}(t, x, y) \leq \mathcal{A}_{R} p_{t \wedge V^{2}(R)}(x-y)\left(\frac{V\left(\delta_{B_{R}}(x)\right)}{\sqrt{t} \wedge V(R)} \wedge 1\right)\left(\frac{V\left(\delta_{B_{R}}(y)\right)}{\sqrt{t} \wedge V(R)} \wedge 1\right) e^{-\lambda_{1} t},
$$

which is the desired uniform upper bound, since finite $\mathcal{A}_{R}$ is nondecreasing with $R$. Integrating the above bound with respect to $y$ over $B_{R}$ we obtain

$$
\mathbb{P}^{x}\left(\tau_{B_{R}}>t\right) \leq \mathcal{A}_{R}\left(\frac{V\left(\delta_{B_{R}}(x)\right)}{\sqrt{t} \wedge V(R)} \wedge 1\right) e^{-\lambda_{1} t} .
$$

Now, we deal with the lower bound. By Lemma 6.8 there are $c_{7}=c_{7}(d)<1, c_{8}=$ $c_{8}(d, \underline{\alpha})$ such that

$$
\begin{aligned}
p_{B_{R}}(t, x, y) \geq & \frac{c_{8}\left(\underline{C}_{R}\right)^{1+d / \underline{\alpha}}\left(C_{R}\right)^{9+d / \underline{\alpha}}}{H_{R}^{2}}\left(\frac{V\left(\delta_{B_{R}}(x)\right)}{\sqrt{t}} \wedge 1\right)\left(\frac{V\left(\delta_{B_{R}}(y)\right)}{\sqrt{t}} \wedge 1\right) \\
& \times\left(p_{t / 2}(0) \wedge t \nu(2|x-y|)\right),
\end{aligned}
$$

provided $0<t \leq c_{7} V^{2}(R) C_{R}$.

Next, using subadditivity of $V$, we observe that

$$
\nu(2|x-y|) \geq \frac{C_{4 R}^{*}}{2^{d+2} V^{2}(|x-y|)|x-y|^{d}} .
$$

Therefore, by the estimate (see Lemma 3.2),

$$
p_{t / 2}(0) \wedge \frac{t}{V^{2}(|x-y|)|x-y|^{d}} \geq c_{9} p_{t}(x-y)
$$

with $c_{9}=c_{9}(d)$, we obtain 


$$
\begin{aligned}
p_{B_{R}}(t, x, y) \geq & \frac{c_{8}\left(\underline{C}_{R}\right)^{1+d / \underline{\alpha}}\left(C_{R}\right)^{9+d / \underline{\alpha}}}{H_{R}^{2}} c_{9}\left(1 \wedge \frac{C_{4 R}^{*}}{2^{d+2}}\right)\left(\frac{V\left(\delta_{B_{R}}(x)\right)}{\sqrt{t}} \wedge 1\right) \\
& \times\left(\frac{V\left(\delta_{B_{R}}(y)\right)}{\sqrt{t}} \wedge 1\right) p_{t}(x-y),
\end{aligned}
$$

provided $0<t \leq c_{7} V^{2}(R) C_{R}$. Applying (42) we have

$p_{B_{R}}(t, x, y) \geq \frac{c_{8}\left(\underline{C}_{R}\right)^{1+d / \underline{\alpha}}\left(C_{R}\right)^{9+d / \underline{\alpha}}}{\left(c_{5} H_{R}\right)^{2}} c_{9}\left(1 \wedge \frac{C_{4 R}^{*}}{2^{d+2}}\right) \mathbb{P}^{x}\left(\tau_{B_{R}}>t\right) \mathbb{P}^{y}\left(\tau_{B_{R}}>t\right) p_{t}(x-y)$.

In particular, taking $t_{0}=c_{7} V^{2}(R) C_{R} \leq V^{2}(R)$, we have

$$
\begin{aligned}
p_{B_{R}}\left(t_{0} / 2, x, y\right) \geq & \frac{c_{8}\left(\underline{C}_{R}\right)^{1+d / \underline{\alpha}}\left(C_{R}\right)^{9+d / \underline{\alpha}}}{\left(c_{5} H_{R}\right)^{2}} c_{9}\left(1 \wedge \frac{C_{4 R}^{*}}{2^{d+2}}\right) p_{t_{0} / 2}(2 R) \\
& \times \mathbb{P}^{x}\left(\tau_{B_{R}}>t_{0} / 2\right) \mathbb{P}^{y}\left(\tau_{B_{R}}>t_{0} / 2\right) .
\end{aligned}
$$

To extend the estimate (44) to $t \geq t_{0}$ we apply Lemma 6.9 with

$$
c^{*}=\frac{c_{8}\left(\underline{C}_{R}\right)^{1+d / \underline{\alpha}}\left(C_{R}\right)^{9+d / \underline{\alpha}}}{\left(c_{5} H_{R}\right)^{2}} c_{9}\left(1 \wedge \frac{C_{4 R}^{*}}{2^{d+2}}\right) p_{t_{0} / 2}(2 R) .
$$

Hence, for $t \geq t_{0}$ we have

$$
p_{B_{R}}(t, x, y) \geq\left(\frac{c^{*}}{\sqrt{\left|B_{R}\right|} p_{t_{0} / 2}(0)}\right)^{2} e^{-\lambda_{1} t_{0}} \mathbb{P}^{x}\left(\tau_{B_{R}}>\frac{t_{0}}{2}\right) \mathbb{P}^{y}\left(\tau_{B_{R}}>\frac{t_{0}}{2}\right) e^{-\lambda_{1} t} .
$$

Next, by Lemma 3.1, there are constants $c_{10}=c_{10}(d), c_{11}=c_{11}(d)$ such that

$$
p_{t_{0} / 2}(2 R) \geq c_{10} t_{0} \nu(2 R) \exp \left(\frac{-c_{11} t_{0}}{V^{2}(2 R)}\right) .
$$

Since $\nu(2 R) \geq \frac{C_{2 R}^{*}}{(2 R)^{d} V^{2}(2 R)}$ by monotonicity and subadditivity of $V$,

$$
\frac{c_{7} C_{R}}{4}=\frac{t_{0}}{4 V^{2}(R)} \leq \frac{t_{0}}{V^{2}(2 R)} \leq c_{7} C_{R} \leq 1,
$$

so there are $c_{12}=c_{12}(d)$ such that

$$
p_{t_{0} / 2}(2 R) \geq c_{12} \frac{C_{2 R}^{*} C_{R}}{R^{d}} .
$$

By Corolarry 6.4 with $r=(1 / 2) c_{7} C_{R}, p_{t_{0} / 2}(0) \leq \frac{c_{13}}{\left(C_{R} \underline{C}_{R}\right)^{1+d / \underline{\alpha}}} \frac{1}{R^{d}}$ with $c_{13}=c_{13}(d, \underline{\alpha})$. This implies that

$$
\left(\frac{p_{t_{0} / 2}(2 R)}{\sqrt{\left|B_{R}\right|} p_{t_{0} / 2}(0)}\right)^{2} \geq c_{14}\left[\left(C_{R} \underline{C}_{R}\right)^{1+d / \underline{\alpha}} C_{2 R}^{*} C_{R}\right]^{2} \frac{1}{R^{d}}
$$

with $c_{14}=c_{14}(d, \underline{\alpha})$.

On the other hand for all $t \geq t_{0}$,

$$
p_{t \wedge V^{2}(R)}(x-y) \leq p_{t_{0} / 2}(0) \leq \frac{c_{13}}{\left(C_{R} \underline{C}_{R}\right)^{1+d / \underline{\alpha}}} \frac{1}{R^{d}}
$$

Combining (46) and (47) we obtain for $t \geq t_{0}$, 


$$
\left(\frac{p_{t_{0} / 2}(2 R)}{\sqrt{\left|B_{R}\right|} p_{t_{0} / 2}(0)}\right)^{2} \geq c_{15}\left(C_{2 R}^{*} C_{R}\right)^{2}\left(C_{R} \underline{C}_{R}\right)^{3+3 d / \underline{\alpha}} p_{t \wedge V^{2}(R)}(x-y)
$$

with $c_{15}=c_{15}(d, \underline{\alpha})$.

Note also that $\lambda_{1} t_{0}=\lambda_{1} V^{2}(R) c_{7} C_{R} \leq c$, where $c=c(d, \underline{\alpha})$. Hence, by (45) and (48), for $t \geq t_{0}$ we have

$$
\begin{aligned}
p_{B_{R}}(t, x, y) \geq & \frac{c_{16}\left(C_{2 R}^{*}\right)^{2} \underline{C}_{R}^{5+5 d / \underline{\alpha}}\left(C_{R}\right)^{23+5 d / \underline{\alpha}}}{H_{R}^{4}}\left(1 \wedge \frac{C_{4 R}^{*}}{2^{d+2}}\right)^{2} \\
& \times \mathbb{P}^{x}\left(\tau_{B_{R}}>\frac{t_{0}}{2}\right) \mathbb{P}^{y}\left(\tau_{B_{R}}>\frac{t_{0}}{2}\right) e^{-\lambda_{1} t} p_{t \wedge V^{2}(R)}(x-y)
\end{aligned}
$$

with $c_{16}=c_{16}(d, \underline{\alpha})$. Due to Lemma $\underline{6.5}$, and since $\mathcal{J}_{R} \geq c(d) C_{2 R}^{*}$, we have the lower bound

$$
\mathbb{P}^{x}\left(\tau_{B_{R}}>t\right) \geq c_{17} \frac{C_{2 R}^{*}}{H_{R}}\left(\frac{V\left(\delta_{B_{R}}(x)\right)}{\sqrt{t}} \wedge 1\right)
$$

for $t \leq c_{18} V^{2}(R)$ with $c_{17}=c_{17}(d)$ and $c_{18}=c_{18}(d)$. Recall that $t_{0}=c_{7} V^{2}(R) C_{R} \leq$ $c_{7} V^{2}(R)$. We may assume that the constant $c_{7}$ is smaller than $c_{18}$. Hence

$$
\mathbb{P}^{x}\left(\tau_{B_{R}}>t_{0}\right) \geq c_{17} \frac{C_{2 R}^{*}}{H_{R}}\left(\frac{V\left(\delta_{B_{R}}(x)\right)}{\sqrt{t_{0}}} \wedge 1\right)
$$

which combined with (49) yields for $t \geq t_{0}$,

$$
\begin{aligned}
p_{B_{R}}(t, x, y) \geq & \frac{c_{19}\left(C_{2 R}^{*}\right)^{4} \underline{C}_{R}^{5+5 d / \underline{\alpha}}\left(C_{R}\right)^{23+5 d / \underline{\alpha}}}{H_{R}^{6}}\left(1 \wedge \frac{C_{4 R}^{*}}{2^{d+2}}\right)^{2} p_{t \wedge V^{2}(R)}(x-y) \\
& \times\left(\frac{V\left(\delta_{B_{R}}(x)\right)}{\sqrt{t} \wedge V(R)} \wedge 1\right)\left(\frac{V\left(\delta_{B_{R}}(y)\right)}{\sqrt{t} \wedge V(R)} \wedge 1\right) e^{-\lambda_{1} t}
\end{aligned}
$$

with $c_{19}=c_{19}(d, \underline{\alpha})$. If we set

$$
\mathcal{A}_{R}^{*}=\left(\frac{(\underline{C})^{1+d / \underline{\alpha}}\left(C_{R}\right)^{9+d / \underline{\alpha}}}{H_{R}^{2}} \wedge \frac{\left(C_{2 R}^{*}\right)^{4} \underline{C}_{R}^{5+5 d / \underline{\alpha}}\left(C_{R}\right)^{23+5 d / \underline{\alpha}}}{H_{R}^{6}}\right)\left(1 \wedge \frac{C_{4 R}^{*}}{2^{d+2}}\right)^{2},
$$

then combining (50) with (44) there is $c_{20}=c_{20}(d, \underline{\alpha})$ such that

$$
p_{B_{R}}(t, x, y) \geq c_{20} \mathcal{A}_{R}^{*} p_{t \wedge V^{2}(R)}(x-y)\left(\frac{V\left(\delta_{B_{R}}(x)\right)}{\sqrt{t} \wedge V(R)} \wedge 1\right)\left(\frac{V\left(\delta_{B_{R}}(y)\right)}{\sqrt{t} \wedge V(R)} \wedge 1\right) e^{-\lambda_{1} t}
$$

for $t>0$. It is clear that $\mathcal{A}_{R}^{*}$ is nonincreasing in $R$, so the proof of the lower bound of $p_{B_{R}}$ is completed.

To finish the proof we need to show a lower bound for $\mathbb{P}^{x}\left(\tau_{B_{R}}>t\right)$. By Lemma 6.5 it is clear that it is enough to consider $t \geq c_{21} V^{2}(R)$ for some $c_{21}=c_{21}(d)<1$. Note that $t \wedge V^{2}(R)=c_{22} V^{2}(R)$ for some $c_{21} \leq c_{22} \leq 1$. We have

$$
p_{t \wedge V^{2}(R)}(x-y) \geq p_{c_{22} V^{2}(R)}(2 R) \geq \frac{C_{2 R} c_{22} V^{2}(R)}{V^{2}(2 R)(2 R)^{d}} \geq \frac{c_{21} C_{2 R}}{4(2 R)^{d}} .
$$

Moreover for $|y| \leq R / 2$ and $t \geq t_{0}$,

$$
\left(\frac{V\left(\delta_{B_{R}}(y)\right)}{\sqrt{t} \wedge V(R)} \wedge 1\right) \geq 1 / 2 .
$$


Integrating $p_{B_{R}}(t, x, y)$ over $B_{R / 2}$ with respect to $y$ and applying (51) provides the desired bound for $\mathbb{P}^{x}\left(\tau_{B_{R}}>t\right)$.

Acknowledgements. We thank prof. J. Zabczyk for communicating to us the problem of gradient estimates of the killed semigroup for jump processes.

\section{REFERENCES}

[1] K. Bogdan, T. Grzywny, M. Ryznar, Barriers, exit time and survival probability for unimodal Lévy processes, Probab. Theory Related Fields 162 (2015), no. 1-2, 155-198.

[2] K. Bogdan, T. Grzywny, M. Ryznar, Density and tails of unimodal convolution semigroups, J. Funct. Anal. 266 (2014), no. 6, 3543-3571.

[3] K. Bogdan, T. Grzywny, M. Ryznar, Dirichlet heat kernel for unimodal Lévy processes, Stochastic Process. Appl. 124 (2014), no. 11, 3612-3650.

[4] K. Bogdan, T. Grzywny, M. Ryznar, Heat kernel estimates for the fractional Laplacian with Dirichlet conditions, Ann. Probab. 38 (2010), no. 5, 1901-1923.

[5] R. Carmona, Path integrals for relativistic Schrödinger operators, Lect. Notes in Phys. 345 (1989), 65-92.

[6] Z.-Q. Chen, P. Kim, R. Song, Dirichlet heat kernel estimates for $\Delta^{\alpha / 2}+\Delta^{\beta / 2}$, Illinois J. Math. 54(4) (2012), 1357-1392.

[7] Z.-Q. Chen, P. Kim, R. Song, Dirichlet heat kernel estimates for rotationally symmetric Lévy processes, Proc. Lond. Math. Soc. (3) 109 (2014), no. 1, 90-120.

[8] Z.-Q. Chen, P. Kim, R. Song, Global heat kernel estimate for relativistic stable processes in exterior open sets, J. Funct. Anal. 263 (2) (2012) 448-475.

[9] Z.-Q. Chen, P. Kim, R. Song, Heat kernel estimates for Dirichlet fractional Laplacian J. Eur. Math. Soc. 12 (2010), 1307-1329.

[10] G. Da Prato, B. Goldys, J. Zabczyk, Ornstein-Uhlenbeck semigroups in open sets of Hilbert spaces, C. R. Acad. Sci. Paris Sr. I Math. 325 (1997), no. 4, 433-438.

[11] S. Fornaro, G. Metafune, E. Priola, Gradient estimates for Dirichlet parabolic problems in unbounded domains, J. Differential Equations 205 (2004), no. 2, 329-353.

[12] T. Grzywny, On Harnack inequality and Hölder regularity for isotropic unimodal Lévy processes, Potential Anal. 41 (2014), no. 1, 1-29.

[13] T. Grzywny, M. Ryznar, B. Trojan, Asymptotic behaviour and estimates of slowly varying convolution semigroups, preprint (2016).

[14] N. Ikeda, S. Watanabe, On some relations between the harmonic measure and the Levy measure for a certain class of Markov processes, J. Math. Kyoto Univ. 2 (1962), 79-95.

[15] K. Kaleta, P. Sztonyk, Estimates of transition densities and their derivatives for jump Lévy processes, J. Math. Anal. Appl. 431 (2015), no. 1, 260-282.

[16] R.Z. Khasminskii, Stochastic stability of differential equations, Sijthoff and Noordhoff, Alphen aan den Rijn, The Netherlands (1980).

[17] P. Kim, R. Song, Z. Vondracek, Global uniform boundary Harnack principle with explicit decay rate and its application, Stochastic Process. Appl. 124 (2014), no. 1, 235-267.

[18] V. Knopova, R. Schilling, A note on the existence of transition probability densities of Lévy processes, Forum Math. 25 (2013), no. 1, 125-149.

[19] T. Kulczycki, Gradient estimates of q-harmonic functions of fractional Schrödinger operator, Potential Anal. 39 (2013), 69-98.

[20] T. Kulczycki, M. Ryznar, Gradient estimates of harmonic functions and transition densities for Lévy processes, Trans. Amer. Math. Soc. 368 (2016), no. 1, 281-318.

[21] T. Kulczycki, B. Siudeja, Intrinsic ultracontractivity of the Feynman-Kac semigroup for relativistic stable processes, Trans. Amer. Math. Soc. 358 (2006), no. 11, 5025-5057.

[22] M. Kwaśnicki, J. Małecki, M. Ryznar, Suprema of Lévy processes, Ann. Prob., 41 (2013), 2047-2065.

[23] E. H. Lieb, R. Seiringer, The stability of matter in quantum mechanics, Cambridge University Press, Cambridge, (2010). 
[24] A. Lunardi, Analytic semigroups and optimal regularity in parabolic problems, Progress in Nonlinear Differential Equations and their Applications, 16; Birkhuser Verlag, Basel, (1995).

[25] M. Majka, Coupling and exponential ergodicity for stochastic differential equations driven by Lévy processes, arXiv:1509.08816

[26] P. W. Millar, First passage distributions of processes with independent increments, Ann. Probability 3 (1975), 215-233.

[27] E. Priola, J. Zabczyk, Structural properties of semilinear SPDEs driven by cylindrical stable processes, Probab. Theory Related Fields 149 (2011), no. 1-2, 97-137.

[28] M. Ryznar, Estimates of Green function for relativistic $\alpha$-stable process, Potential Anal. 17 (2002), 1-23.

[29] R. Schilling, P. Sztonyk, J. Wang, Coupling property and gradient estimates of Lévy processes via the symbol, Bernoulli 18 (2012), 1128-1149.

[30] M. L. Silverstein, Classification of coharmonic and coinvariant functions for a Lévy process, Ann. Probab. 8 (1980), 539-575.

[31] B. Siudeja, Symmetric stable processes on unbounded domains, Potential Anal. 25 (2006), 371-386.

[32] P. Sztonyk, On harmonic measure for Lévy processes, Probab. Math. Statist. 20 (2000), 383390.

[33] S. R. S. Varadhan, Lectures on Diffusion Problems and Partial Differential Equations, Tata Institute of Fundamental Research, Bombay, (1980).

[34] N. T. Varopoulos, Gaussian estimates in Lipschitz domains, Canad. J. Math. 55 (2003), 401431.

[35] F.-Y. Wang, Gradient estimates of Dirichlet heat semigroups and application to isoperimetric inequalities, Ann. Probab. 32 (2004), no. 1A, 424-440.

[36] T. Watanabe, The isoperimetric inequality for isotropic unimodal Lévy processes, Z. Wahrhrsch. Verw. Gebiete 63 (1983), 487-499.

[37] Q. S. Zhang, Some gradient estimates for the heat equation on domains and for an equation by Perelman, Int. Math. Res. Not. 2006, 1-39.

[38] Q. S. Zhang, The boundary behavior of heat kernels of Dirichlet Laplacians, J. Differential Equations 182 (2002), 416-430.

Faculty of Pure and Applied Mathematics, Wroceaw University of Science and Technology, Wyb. Wyspiańskiego 27, 50-370 Wroceaw, Poland.

E-mail address: Tadeusz.Kulczycki@pwr.edu.pl

E-mail address: Michal.Ryznar@pwr.edu.pl 